\title{
Recent Advances in Heliogyro Solar Sail Structural Dynamics, Stability, and Control Research
}

\author{
W. Keats Wilkie ${ }^{1}$, Jerry E. Warren ${ }^{2}$, Lucas G. Horta ${ }^{3}$, Karen H. Lyle ${ }^{4}$ and Jer-Nan Juang \\ NASA Langley Research Center, Hampton, Virginia 23681-2199 \\ S. Chad Gibbs ${ }^{6}$ and Earl H. Dowell ${ }^{7}$ \\ Duke University, Durham, North Carolina, 27708-0287 \\ Daniel V. Guerrant ${ }^{8}$ and Dale Lawrence ${ }^{9}$ \\ Univeristy of Colorado, Boulder, Colorado 80309-0429
}

\begin{abstract}
Results from recent NASA sponsored research on the structural dynamics, stability, and control characteristics of heliogyro solar sails are summarized. Specific areas under investigation include coupled nonlinear finite element analysis of heliogyro membrane blade with solar radiation pressure effects, system identification of spinning membrane structures, and solarelastic stability analysis of heliogyro solar sails, including stability during blade deployment. Recent results from terrestrial 1-g blade dynamics and control experiments on "rope ladder" membrane blade analogs, and small-scale in vacuo system identification experiments with hanging and spinning high-aspect ratio membranes will also be presented. A low-cost, rideshare payload heliogyro technology demonstration mission concept is used as a mission context for these heliogyro structural dynamics and solarelasticity investigations, and is also described. Blade torsional dynamic response and control are also shown to be significantly improved through the use of edge stiffening structural features or inclusion of modest tip masses to increase centrifugal stiffening of the blade structure. An output-only system identification procedure suitable for on-orbit blade dynamics investigations is also developed and validated using ground tests of spinning sub-scale heliogyro blade models. Overall, analytical and experimental investigations to date indicate no intractable stability or control issues for the heliogyro solar sail concept.
\end{abstract}

$\begin{array}{ll}A U & =\text { Astronomical Unit, } 1.49597871 \times 10^{8} \mathrm{~m} \\ P & =\text { nondimensional rotational frequency, } \omega / \Omega \\ a_{c} & =\text { characteristic acceleration at } 1 \mathrm{AU}, \mathrm{mm} / \mathrm{sec}^{2} \\ \omega & =\text { heliogyro blade modal frequency, rad } / \mathrm{sec}^{2} \mathrm{P} \\ \Omega & =\text { nominal rotation rate of heliogyro, } \mathrm{rad} / \mathrm{sec} \text { or } \mathrm{RPM}\end{array}$

\footnotetext{
${ }^{1}$ Branch Head, Structural Dynamics Branch, Mail Stop 230, Senior Member, AIAA.

${ }^{2}$ Senior Research Aerospace Engineer, Structural Dynamics Branch, Mail Stop 230, Senior Member AIAA.

${ }^{3}$ Senior Research Aerospace Engineer, Structural Dynamics Branch, Mail Stop 230, Senior Member AIAA.

${ }^{4}$ Senior Research Aerospace Engineer, Structural Dynamics Branch, Mail Stop 230, Senior Member AIAA.

${ }^{5}$ Distinguished Research Associate, Structural Dynamics Branch, Mail Stop 230, Fellow AIAA.

${ }^{6}$ Ph.D Candidate, Department of Mechanical Engineering and Material Science, Hudson Hall Box 900, Student Member AIAA.

${ }^{7}$ Professor and Chair, Department of Mechanical Engineeringand Material Science, 141 Hudson Hall, Honorary Fellow AIAA.

${ }^{8}$ Ph.D Candidate, Department of Aerospace Engineering Sciences, 429 UCB, Student Member AIAA.

${ }^{9}$ Professor, Department of Aerospace Engineering Sciences, 429 UCB, Associate Fellow AIAA.
} 


\section{Introduction}

$\mathrm{T}$ HE heliogyro is a structurally efficient, high performance, helicopter-like spinning solar sail architecture. ${ }^{1}$ The sail elements of the heliogyro are high aspect ratio reflective membrane strips, or "blades", up to kilometers in length. Blade stiffness is created by centrifugal forces alone. This results in an overall solar sail structure that is exceptionally light weight, and maximizes the acceleration of the vehicle due to solar radiation pressure. A heliogyro concept studied by the Jet Propulsion Laboratory (JPL) in 1977, is shown in Fig. 1.

The sail blades of the heliogyro are stowed and deployed using reels, which simplifies sail packaging and deployment. Six degree-of-freedom attitude control of the entire heliogyro vehicle is performed using blade pitch actuation and solar radiation pressure. Blade root pitch control may be performed collectively to generate torques about the spin axis, or cyclically, i.e., in a per-revolution fashion, to generate thrust components in the plane of rotation. Combinations of collective and cyclic pitch can create overturning moments.

In 2010, the successful launch and flight of the Japan Aerospace Exploration Agency (JAXA) IKAROS solar sail ${ }^{3}$ renewed interest within NASA in solar sailing technology, and spinning solar sail architectures in particular. The heliogyro is now being re-evaluated as a practical, advanced solar sail propulsion system for more difficult low thrust missions. Unfortunately, ground testing of full-scale heliogyro solar sails, with sail blades hundreds to thousands of meters long, is impractical due to gravity. Preflight validation testing of full-scale heliogyro designs must therefore be conducted via analysis, and where possible, ground testing of selected key components or sub-scale dynamic models.

In this paper, we will present results from several recent analytical and experimental heliogyro structural dynamics investigations. Specific topics will include coupled nonlinear finite element analysis of heliogyro membrane blade with solar radiation pressure effects, solarelastic stability analysis of heliogyro solar sails, nonlinear blade control experiments using full-chord-scale, vertically suspended "rope ladder" heliogyro blade analog structures, and in vacuo system identification experiments on small-scale hanging and spinning membrane structures. A low-cost heliogyro technology demonstration mission concept will also be described, and is used as a design reference for these structural dynamics, solarelasticity, and control studies. Selected key results are presented and discussed below.

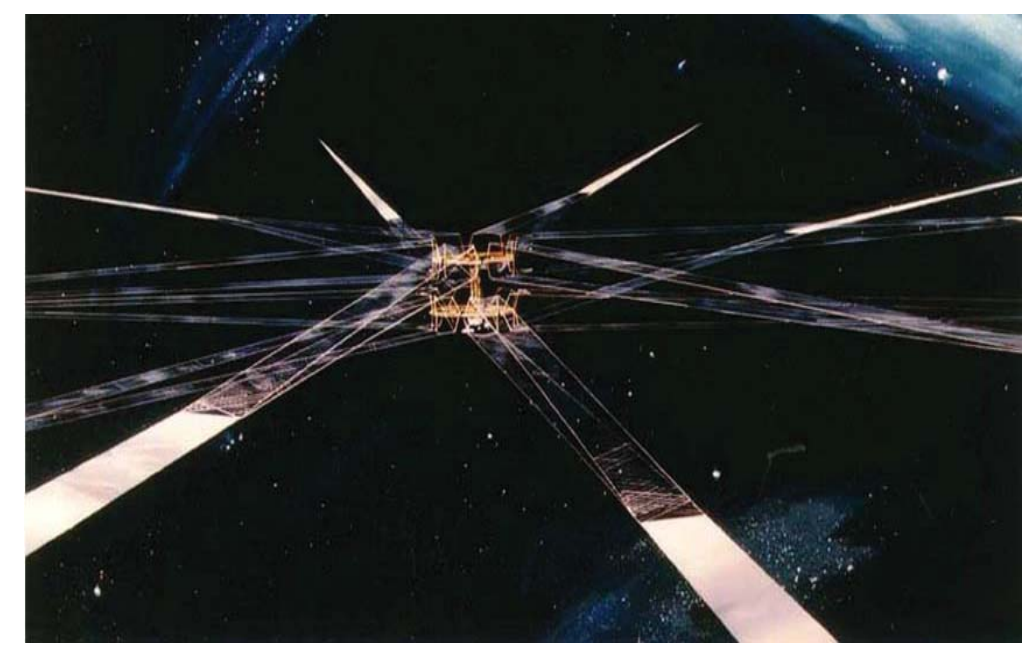

Figure 1. JPL Comet Halley rendezvous heliogyro solar sail concept. Membrane blades are 7.5 km long. ${ }^{2}$

\section{Heliogyro Design Reference Concept}

HELIOS (High-Performance, Enabling, Low-Cost, Innovative, Operational Solar Sail) is our design reference heliogyro concept. ${ }^{4}$ It was developed to provide a near-term mission context for NASA heliogyro technology development activities, including the structural dynamics and stability investigations described in this paper. HELIOS is based upon the 200-m diameter MIT heliogyro concept proposed in $1989,{ }^{5}$ and is designed to be capable of a characteristic acceleration ${ }^{6}, a_{c}$, on the order of $0.5 \mathrm{~mm} / \mathrm{s}^{2}$ at $1.0 \mathrm{AU}$; placing it in the range of solar sail technologies required to enable a variety of science and human exploration support missions. ${ }^{7}$ The HELIOS spacecraft uses modern small-sat and CubeSat technology to minimize bus weight, and is packageable as a secondary payload in order to reduce launch costs. The central core of the HELIOS heliogyro vehicle, with blade reels, blades, and videogrammetry 
camera mast deployed, is shown in Fig. 2. Key HELIOS heliogyro design reference parameters are summarized in Tab. 1.

Rotary stepper motor actuators located at the root of each blade will be used to control blade orientation with respect to the sun, and generate attitude control moments for the HELIOS vehicle. Blade deployment operations will take place at very slow blade deployment rates; on the order of $\mathrm{mm} / \mathrm{sec}$. Controlled collective pitching of the blades will also take place to adjust spin rate during deployment.

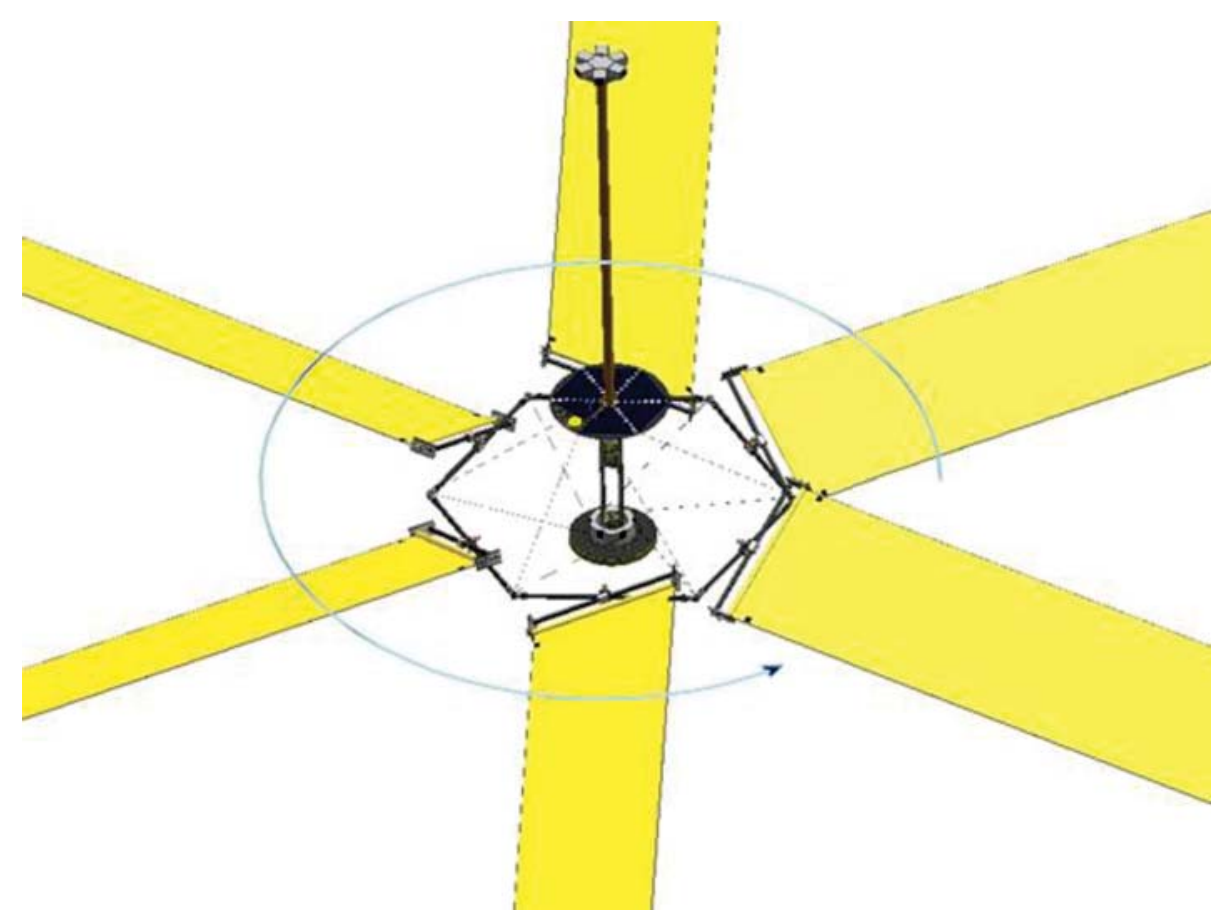

Figure 2. HELIOS heliogyro technology flight demonstration reference concept. Blades at full deployment are $220 \mathrm{~m}$ long. Deployed blade area is $990 \mathrm{~m}^{2}$. Total mass is $18.3 \mathrm{~kg}$.

Table 1. HELIOS Design Reference Heliogyro Parameters

\begin{tabular}{ll}
\hline Property & Value \\
\hline \hline Total sailcraft mass & $18.3 \mathrm{~kg}$ \\
Specular efficiency of blades (-) & 0.85 \\
Sail area at full deployment & $990 \mathrm{~m}^{2}$ \\
Characteristic acceleration, 1 AU & $0.46 \mathrm{~mm} / \mathrm{s}^{2}$ \\
Sail material: & \\
\multicolumn{1}{r}{ Metallization, top and bottom } & $2.54 \mu \mathrm{m}$ Mylar \\
\multicolumn{1}{l}{ Mentral bus mass } & $5 \mathrm{~kg}$ aluminum \\
Sail deployment system mass & $8.3 \mathrm{~kg}$ \\
Sail blade mass (6 blades) & $5 \mathrm{~kg}$ \\
Blade chord & $0.75 \mathrm{~m}$ \\
Blade length, full deployment & $220 \mathrm{~m}$ \\
Rotational period & $1 \mathrm{minute}$ \\
Blade root stress & $4.89 \times 10^{5} \mathrm{~Pa}$ \\
Blade root allowable stress & $55 \times 10^{6} \mathrm{~Pa}$ \\
Blade root tension load & $1.0 \mathrm{~N}$ \\
\hline
\end{tabular}

American Institute of Aeronautics and Astronautics 
Deployment will be paused periodically to evaluate overall spin balance, structural dynamics, and flight control characteristics. This will be performed using bus sensors and a dedicated camera system positioned above the plane of rotation by a deployable mast. Camera imagery will be stored and periodically downlinked for analysis on the ground. As noted by MacNeal in his original studies, the thin membrane blades of the heliogyro possess near-zero damping in both the out-of-plane and torsion directions. ${ }^{1,8}$ Blade damping for HELIOS will be augmented using sun angle information from distributed photovoltaic sensors along the blades, and closed-loop actuation of the pitch control motors. As rotation rates are very low; on the order of 1 RPM; blade dynamic time scales will be very long and easily within the control bandwidth of the blade root pitch motors.

\section{Recent Heliogyro Structural Dynamics and Controls Results}

\section{A. Heliogyro Blade Structural Dynamics Simulations}

As full-scale ground testing of the HELIOS heliogyro is impossible, validation of the HELIOS design will need to be performed analytically. High-fidelity, fully-coupled structural dynamic simulations of the HELIOS vehicle will need to be developed to accomplish this. These models will be used to simulate flight structural dynamics experiments and to help validate simpler, more numerically efficient heliogyro dynamics analysis tools. For our high-fidelity heliogyro simulations, we are using the nonlinear modeling capabilities of the Abaqus commercial finite element code. ${ }^{9}$

Fully-coupled HELIOS blade structural dynamics finite element based simulations including solar radiation pressure have been performed using Abaqus. Two detailed example cases for a single freely-spinning heliogyro blade subjected to $1 \mathrm{AU}$ solar radiation pressure are described below. These cases illustrate predicted blade dynamic behavior for two blade root pitch command profiles that would be used during an operational heliogyro mission. The first root pitch profile is a linear ramp from $0^{\circ}$ to $-25^{\circ}$. This profile is intended to simulate a collective pitch maneuver that would change the thrust and associated acceleration of the sailcraft. The second pitch profile is a once-perrevolution (1P) $\pm 25^{\circ}$ cyclic pitch. This pitch profile would be used to alter the attitude of the spacecraft.

1. Finite Element Model

An Abaqus finite element model was used to conduct nonlinear dynamic analyses of a single spinning heliogyro blade. A visual representation of the finite element model is included in Fig. 3. The heliogyro blade was modeled as a $2.74 \mu \mathrm{m}$ thick uniform membrane using quadrilateral membrane elements with a typical element size equal to $1.0 \mathrm{~m}$ long by $0.1875 \mathrm{~m}$ wide. The material properties for the blade are also shown in Fig. 3.

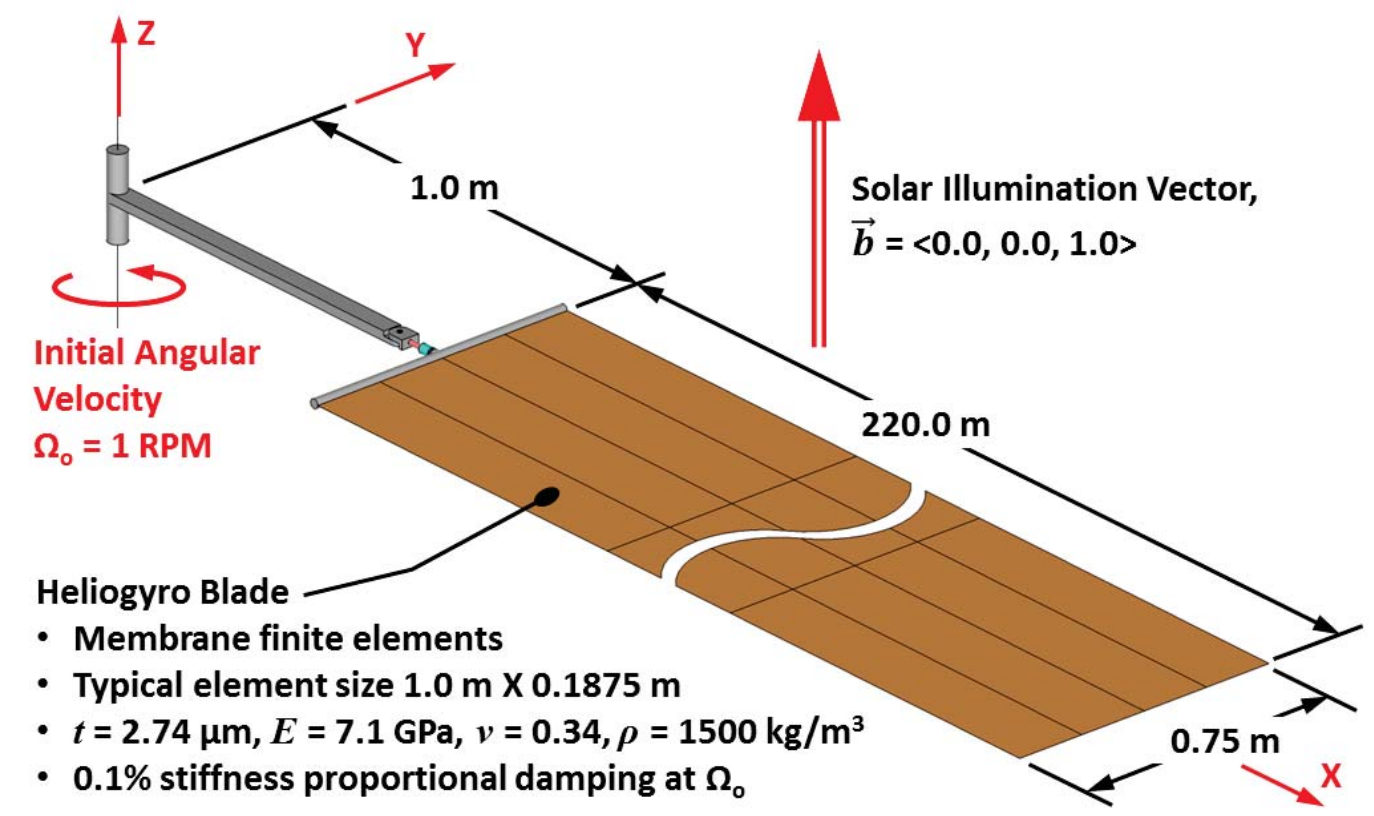

Figure 3. Heliogyro blade finite element model details. 


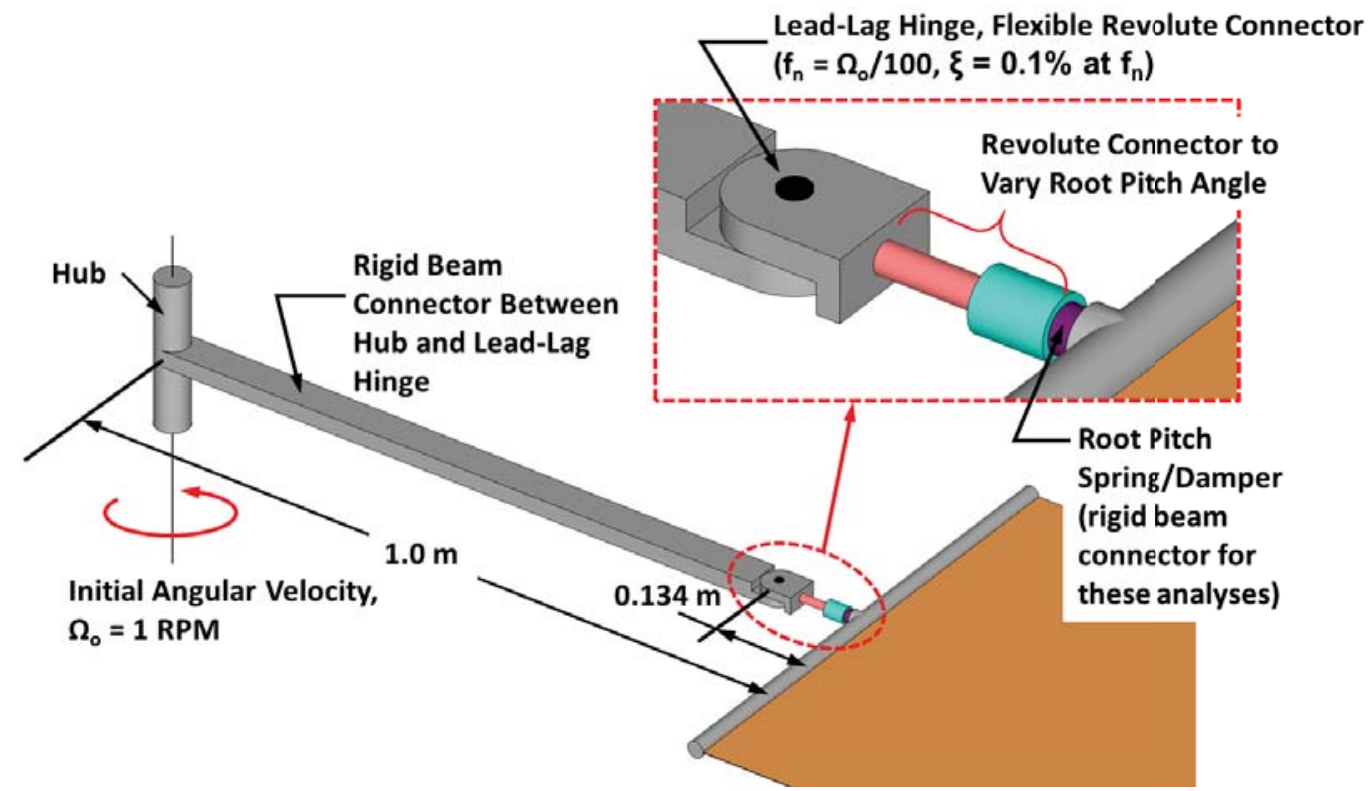

Figure 4. Heliogyro blade root mechanisms.

The heliogyro blade was attached to the hub using a series of root mechanisms. The configuration of the root mechanisms and associated connector elements is shown in Fig. 4. A rigid beam-type connector element was used between the hub and the lead-lag hinge. The lead-lag hinge was modeled using a flexible revolute connector element. The stiffness of the lead-lag hinge was chosen so that the resulting natural frequency of the hinged portion of the model would be one-hundredth of the initial angular velocity. This guarantees that the frequency of the hinged portion of the model will be small compared to the first structural mode of the blade which occurs near the initial angular velocity of the blade. Damping for the lead-lag hinge was chosen to be $0.1 \%$ of critical damping at the hinge frequency. A second rigid revolute connector element was used to prescribe pitch inputs at the root of the blade. The model also included provisions for a root pitch spring/damper between the root pitch input connector and the root of the blade. This spring/damper, which could be used to passively control blade-tip twist oscillations, was modeled as a rigid beam-type connector.

\section{Finite Element Analysis Steps}

Implicit nonlinear dynamic analyses were divided into four steps to predict the response of the heliogyro blade response due to two root pitch command profiles. For convenience the durations of the analysis steps are also shown in parentheses as the approximate number of blade revolutions (revs) based on the 1.0 RPM initial angular velocity initial conditions. The first three steps were the same for both analyses, and were used to apply the appropriate initial conditions and loads to the models prior to the root pitch profiles. The first analysis step allowed the blade to spin freely for 240 seconds (4 revs) under the influence of an initial angular velocity condition of 1.0 RPM. The purpose of this step is to properly tension the heliogyro blade and to allow any artificially induced dynamic response to settle before subsequent loads are introduced into the analysis. The second analysis step included a nominal 1 AU solar radiation pressure $\left(p_{0}\right)$ equal to 9.0E-06 Pa with the solar illumination vector $(\vec{b})$ directed along the positive $\mathrm{Z}$ axis as shown in Fig. 3. The solar radiation pressure was applied to the model as element pressure loads over 240 seconds (4 revs). Individual element pressure loads were calculated in real-time using the external subroutine capability available in Abaqus ${ }^{\mathrm{TM}}$. An external user element subroutine was used to monitor element nodal displacements and to compute the corresponding element normal vectors $(\vec{n})$. The element normal vectors were used to compute orientation specific element solar radiation pressure loads using the standard expression:

$$
\mathrm{p}_{\mathrm{E}}=\mathrm{p}_{0} \times(\overrightarrow{\mathrm{b}} \cdot \overrightarrow{\mathrm{n}})^{2}
$$

Element solar radiation pressures were automatically updated every time the element stiffness matrices were updated, and were applied to the heliogyro blade using an external user distributed load subroutine. In the third analysis step the blade was allowed to spin freely for 1,200 seconds (20 revs) to allow the dynamics caused by

American Institute of Aeronautics and Astronautics 
application of the solar pressure load to settle. The fourth step included different root pitch profiles for each analysis. Blade root pitch command profiles for both example cases are shown in Fig. 5. The first analysis, Case 1, included a linear ramp pitch profile from $0^{\circ}$ to $-25^{\circ}$ over 240 seconds ( 4 revs) as shown. The free-spinning blade was then allowed to respond freely for an additional 1,560 seconds (26 revs). The second analysis, Case 2, included a onceper-revolution (1P) $\pm 25^{\circ}$ cyclic root pitch profile that was linearly ramped over 240 seconds ( 4 revs). The $\pm 25^{\circ}$ cyclic root pitch was applied to the blade for an additional 1,560 seconds (26 revs). The response from the two analysis cases is discussed in the following section.

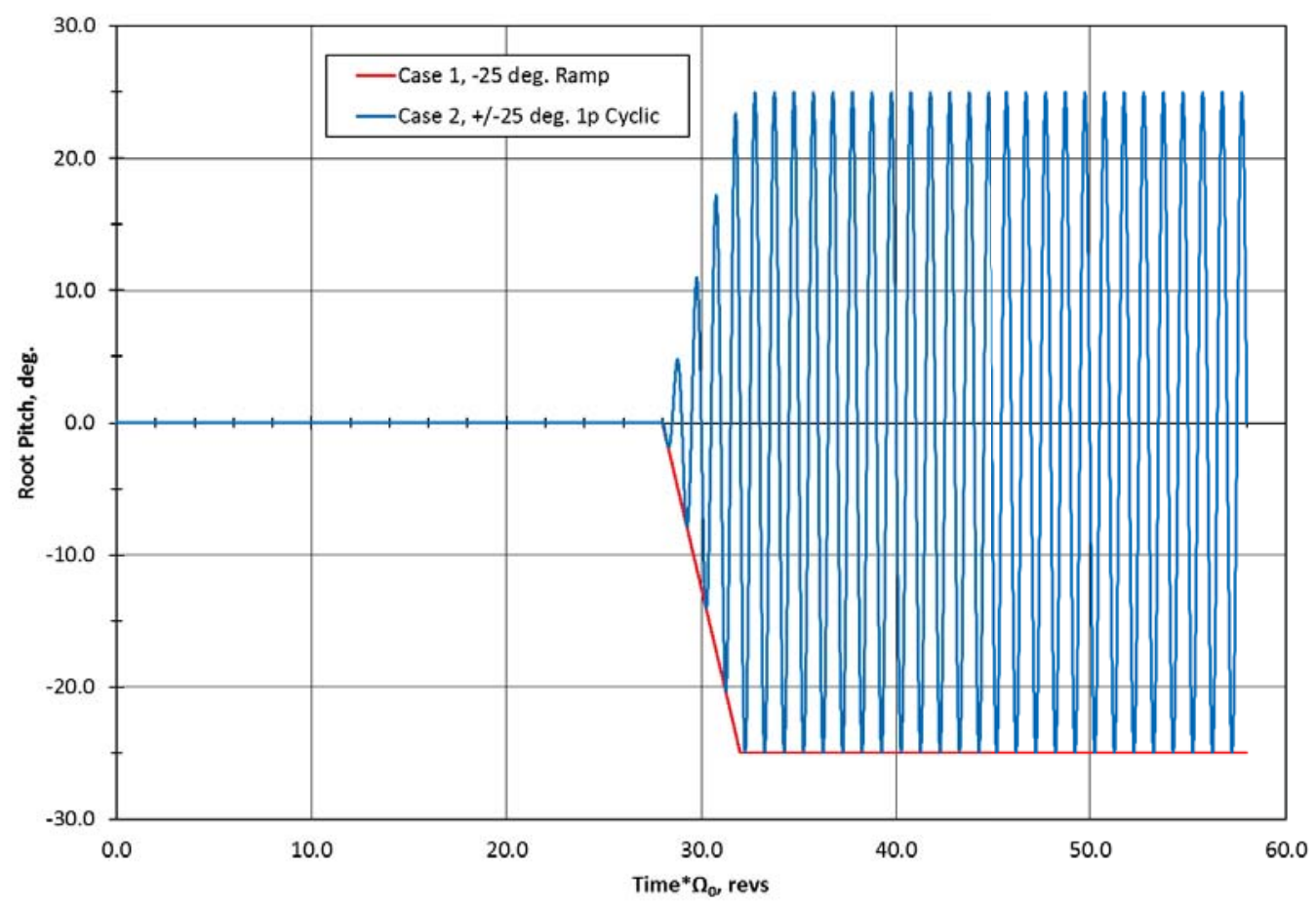

Figure 5. Blade root pitch input profiles for analysis Case 1 (-25 degree collective pitch input) and analysis Case 2 (25 degree 1P cyclic pitch input).

\section{Finite Element Analysis Results and Discussion}

Displacement time histories and normalized hub angular velocity histories for Case 1 ( $\left.-25^{\circ} \mathrm{ramp}\right)$ and Case 2 ( $\pm 25^{\circ}$ cyclic pitch) were generated using the analysis steps described above. The displacement time histories are measured relative to the XYZ coordinate system, shown in Figure 1, which is rotating with the blade. The following terminology is used when describing results for the various directions: $\mathrm{X}$ is longitudinal, $\mathrm{Y}$ is inplane, $\mathrm{Z}$ is flap, and twist is rotation about the $\mathrm{X}$-axis. The hub angular velocities are measured relative to the fixed global coordinate system and then normalized by the 1.0 RPM initial angular velocity of the blade. Results were recovered at a $4 \mathrm{~Hz}$ sample rate and contained a lot of extraneous high frequency information. Therefore, all time history results were filtered forward then backward using an 8th order Butterworth filter with a $0.2 \mathrm{~Hz}$ cutoff frequency. This guarantees that all relevant data below 10 RPM is preserved. For convenience the time axis in all of the following plots was converted to an approximate number of blade revolutions based on the 1.0 RPM initial angular velocity initial conditions.

Results for Case 1 are presented in Figs. 6 through 10. Figure 6 contains a plot of normalized hub angular velocity. As expected, the angular velocity increases in response to the $-25^{\circ}$ degree root pitch because the resultant solar radiation force component along the $\mathrm{Y}$-axis acts in the same direction that the blade is spinning. If the blade were pitched in the opposite direction the angular velocity would decrease. Figure 7 contains a plot of root pitch and the resulting tip twist time history. The resulting average tip twist is significantly less than the root pitch and the tip twist is oscillatory even though the root pitch was applied over 4 revs. A plot of tip flap displacement is included in Fig. 8. Prior to the $-25^{\circ}$ degree root pitch the solar radiation pressure causes the blade tip to displace approximately 275 millimeters. Beyond 28 revs the tip displacement decreases. This is partially due to the decrease in pressure loading

American Institute of Aeronautics and Astronautics 
caused by the blade twist, and partially due to the increasing centrifugal forces as the blade and hub rotation rate increases. A plot of tip inplane displacement is shown in Fig. 9. Beyond 28 revs the blade twist and incident solar radiation pressure combine to produce an average inplane tip displacement component approximately equal to 35 millimeters. Figure 10 contains a plot of tip longitudinal displacement. Prior to the $-25^{\circ}$ degree root pitch the average tip deflection is approximately 8.1 millimeters. After 28 revs the blade elongates due to the increase in angular velocity caused by the $-25^{\circ}$ degree root pitch.

Results for Case 2 are presented in Figs. 11 through 15. A plot of normalized hub angular velocity is included in Fig. 11. The angular velocity does oscillate by a small amount in response to the $\pm 25^{\circ}$ cyclic root pitch, but the average angular velocity remains nearly constant for the duration of the analysis. Figure 12 includes a plot of root pitch and the corresponding tip twist. The tip twist is in phase with the $\pm 25^{\circ}$ cyclic root pitch, but the tip twist amplitude is as much as 68 percent larger than the root pitch amplitude. It is also interesting to note that this behavior continues for the duration of the analysis because the structural damping is very small. A plot of tip flap displacement is shown in Fig. 13. Beyond 28 revs the tip flap displacement oscillates in response to the blade twist oscillations. The maximum displacement is always normal to the surface of the blade, therefore as the blade twists the component of displacement in the original unrotated flap direction decreases. The $\pm 25^{\circ}$ cyclic root pitch reduces the average flap displacement by approximately 20 percent. Figure 14 contains a plot of tip inplane displacement. Similar to the tip flap displacement, the tip inplane displacement oscillates in response to the blade twist oscillations. As the blade twists the component of displacement in the original unrotated inplane direction increases/decreases accordingly. A plot of tip longitudinal displacement is shown in Fig. 15. The tip longitudinal displacement also oscillates in response to the $\pm 25^{\circ}$ cyclic root pitch. The component of displacement in the original unrotated longitudinal direction increases as the blade flap decreases. The average tip longitudinal displacement only increases approximately 0.5 percent due to the $\pm 25^{\circ}$ cyclic pitch.

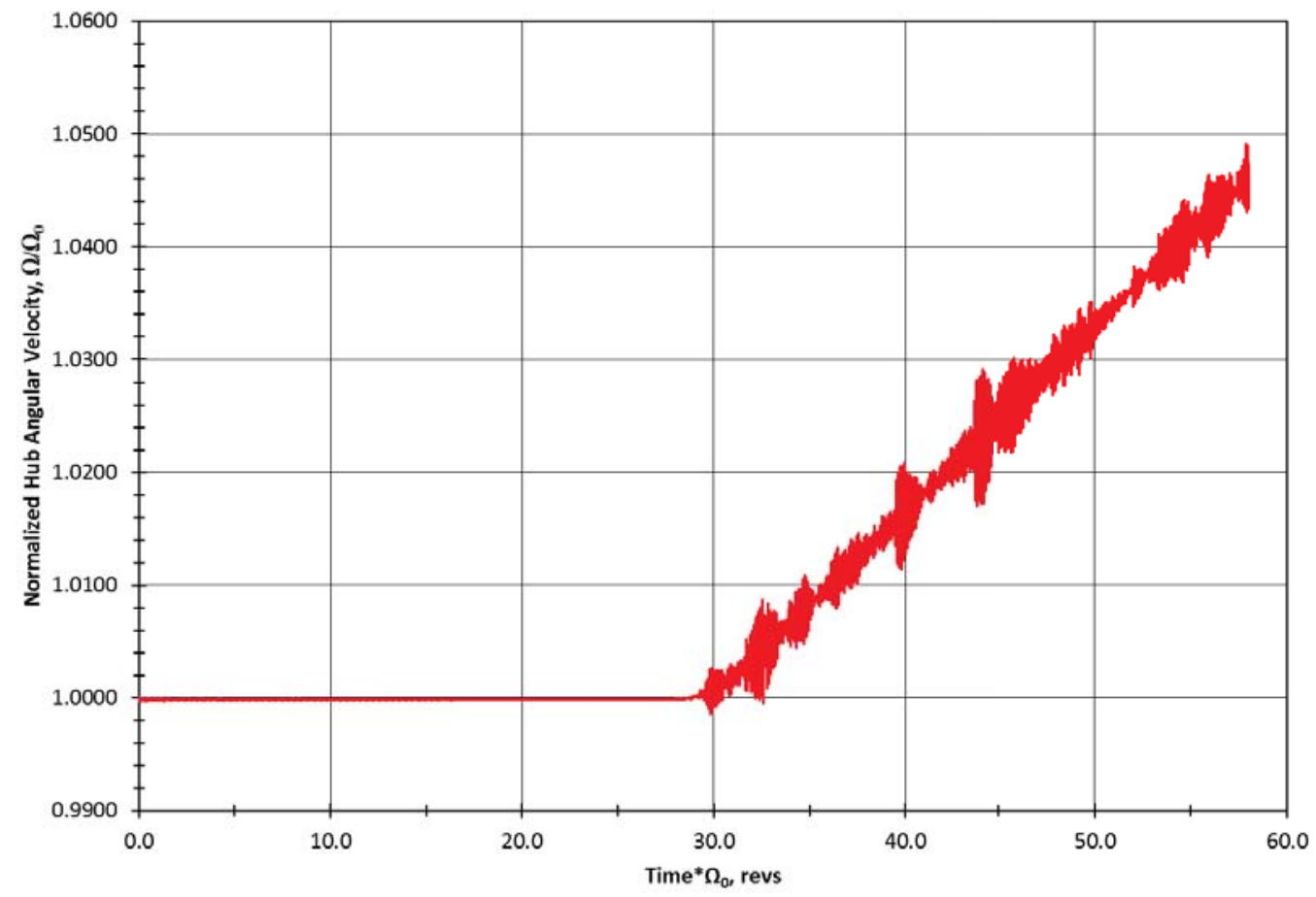

Figure 6. Case 1, -25 degree collective pitch input: Normalized hub angular velocity. 


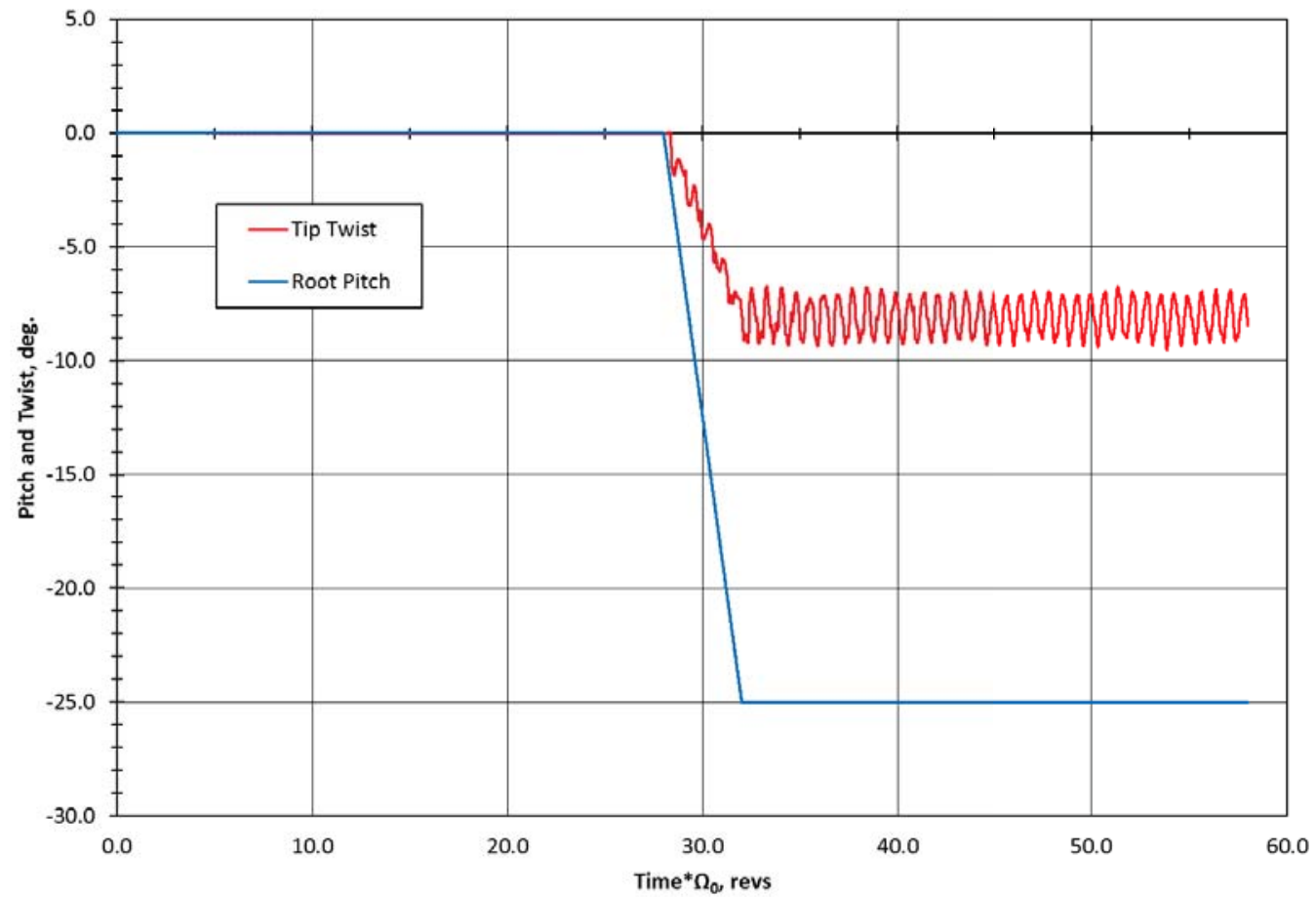

Figure 7. Case 1, -25 degree collective pitch input: Blade root pitch input and tip twist.

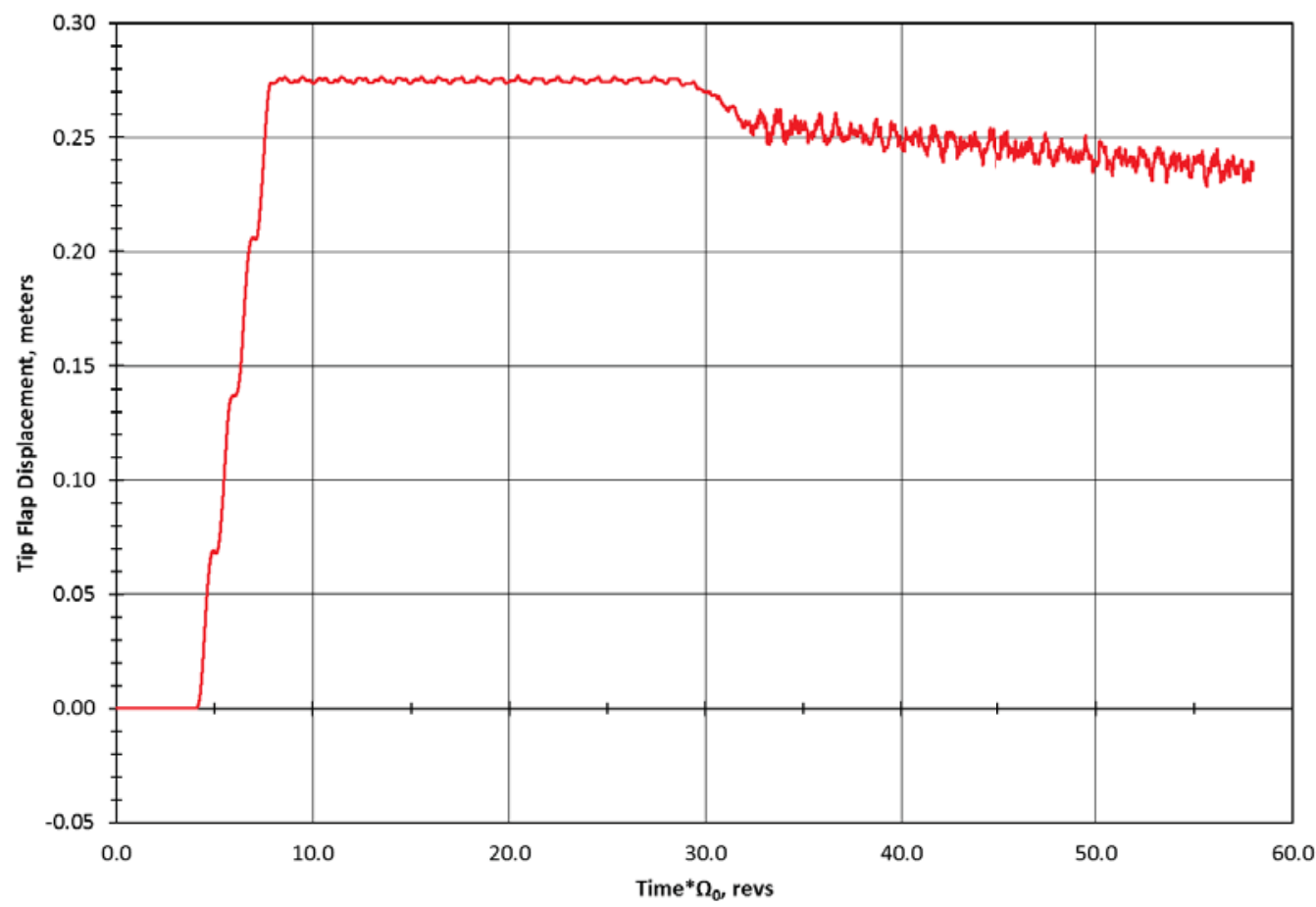

Figure 8. Case 1, -25 degree collective pitch input: Blade tip out-of-plane displacement. 


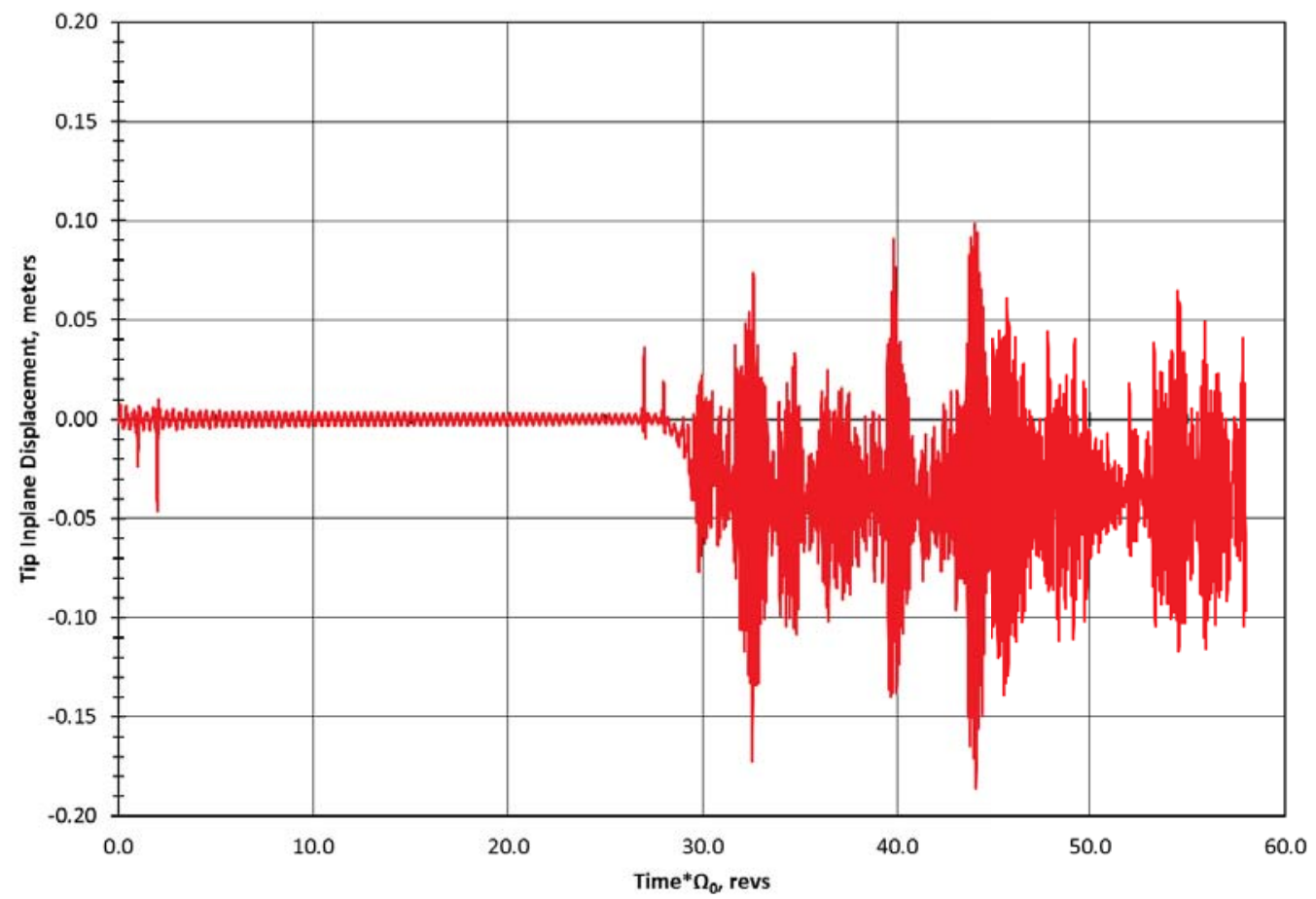

Figure 9. Case 1, -25 degree collective pitch input: Blade tip in-plane displacement.

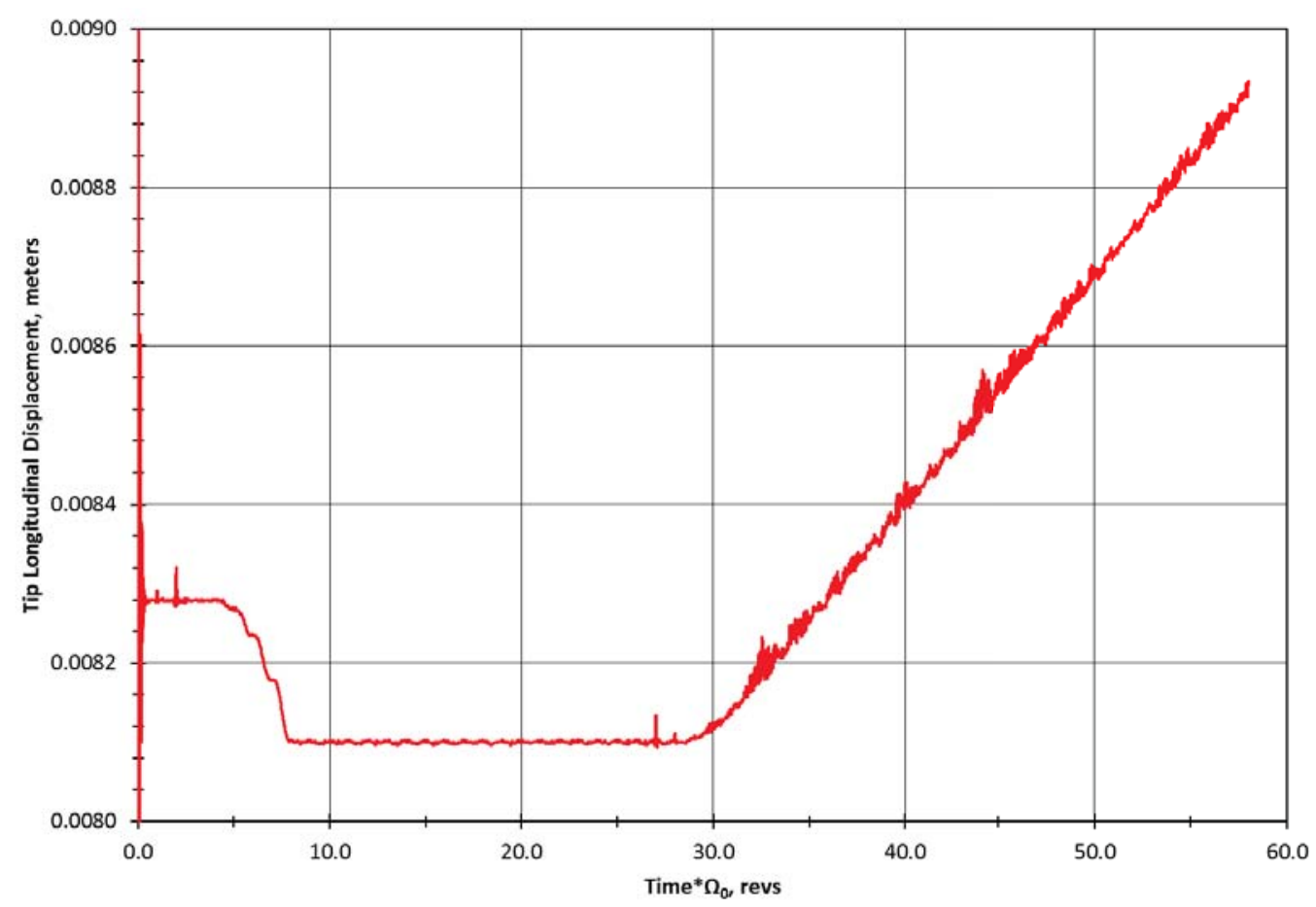

Figure 10. Case 1, -25 degree collective pitch input: Blade tip axial displacement. 


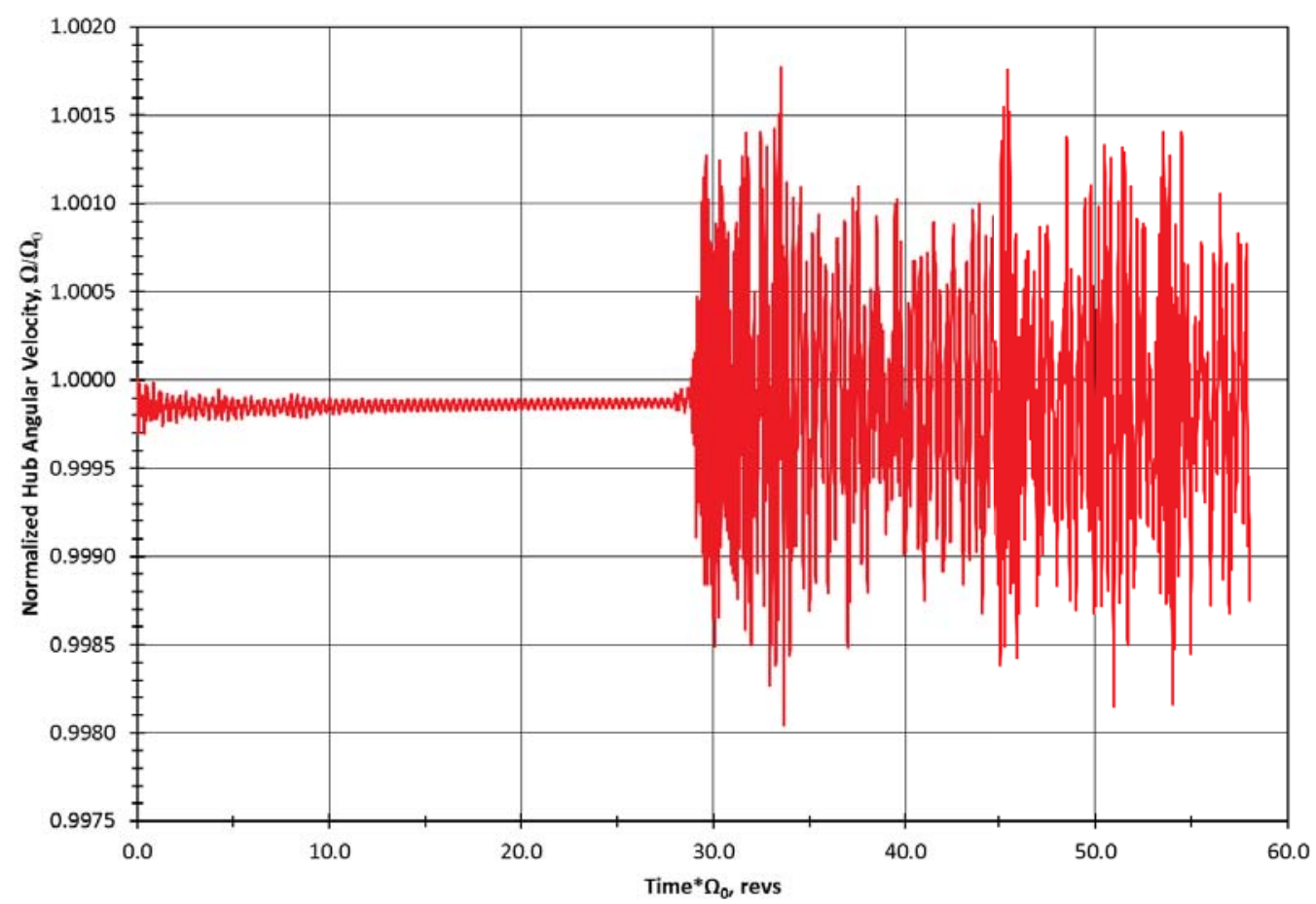

Figure 11. Case 2, 25 degree 1P cyclic pitch input: Normalized hub angular velocity.

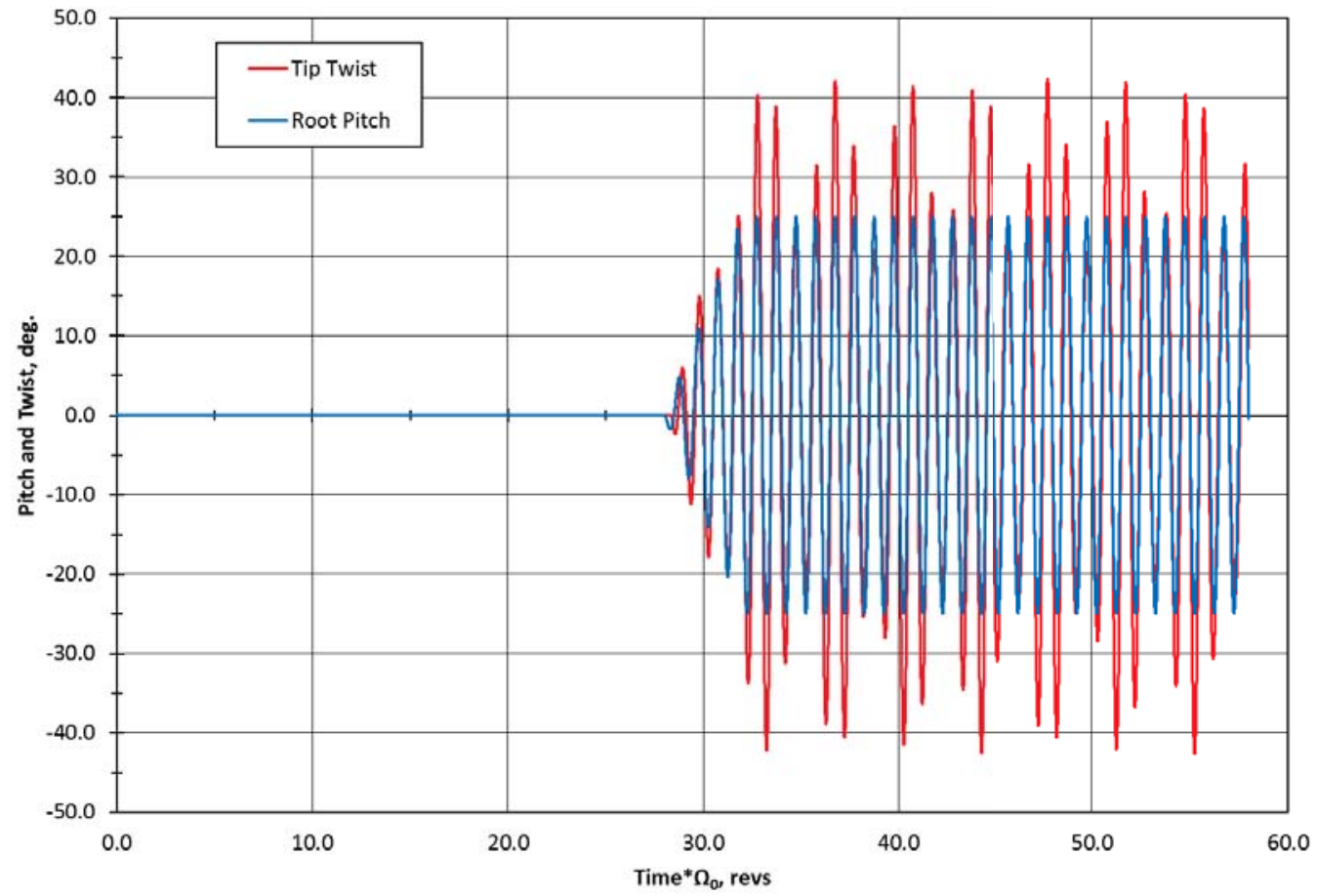

Figure 12. Case 2, 25 degree 1P cyclic pitch input: Blade root pitch input and tip twist. 


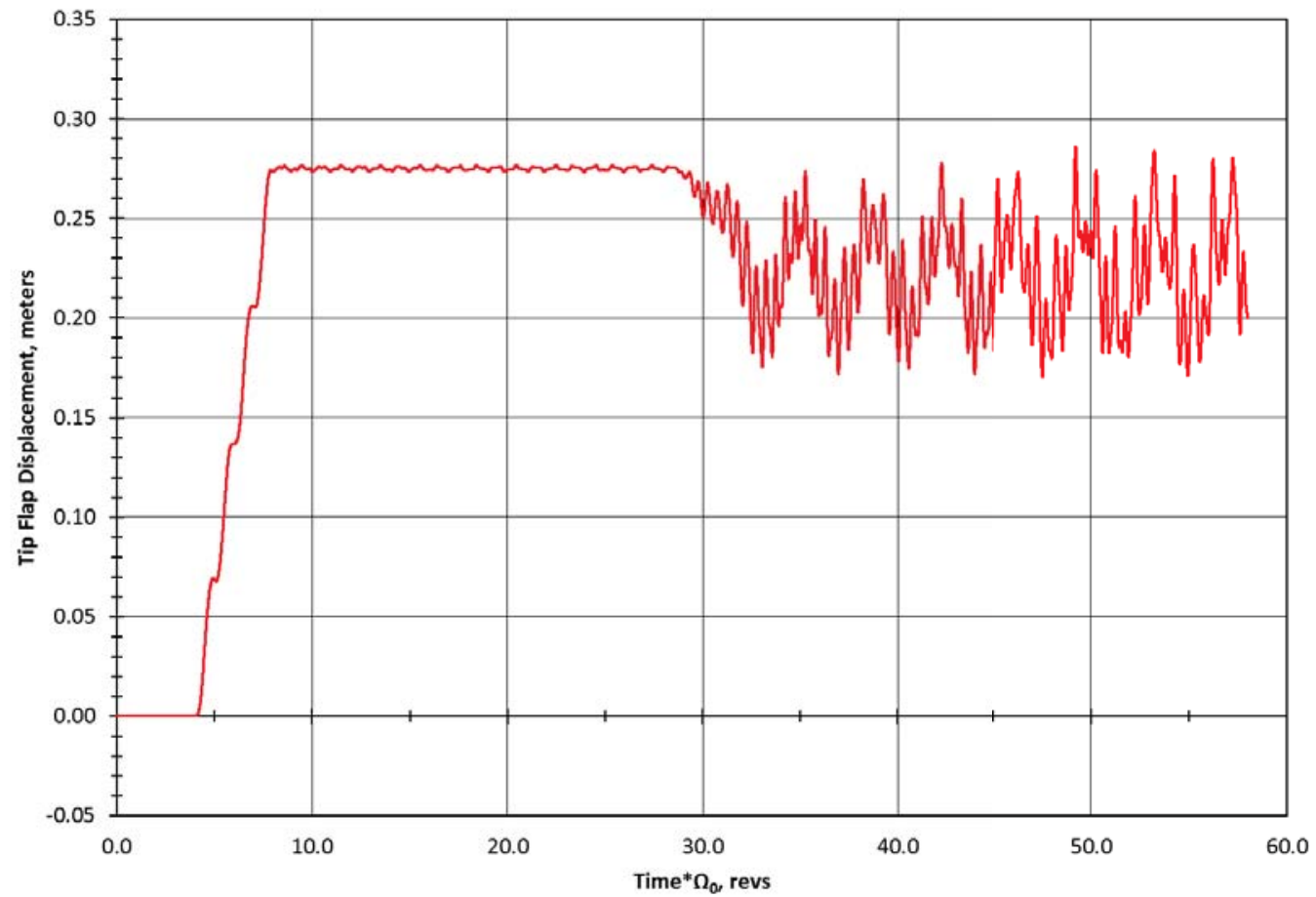

Figure 13. Case 2, 25 degree 1P cyclic pitch input: Blade tip out-of-plane displacement.

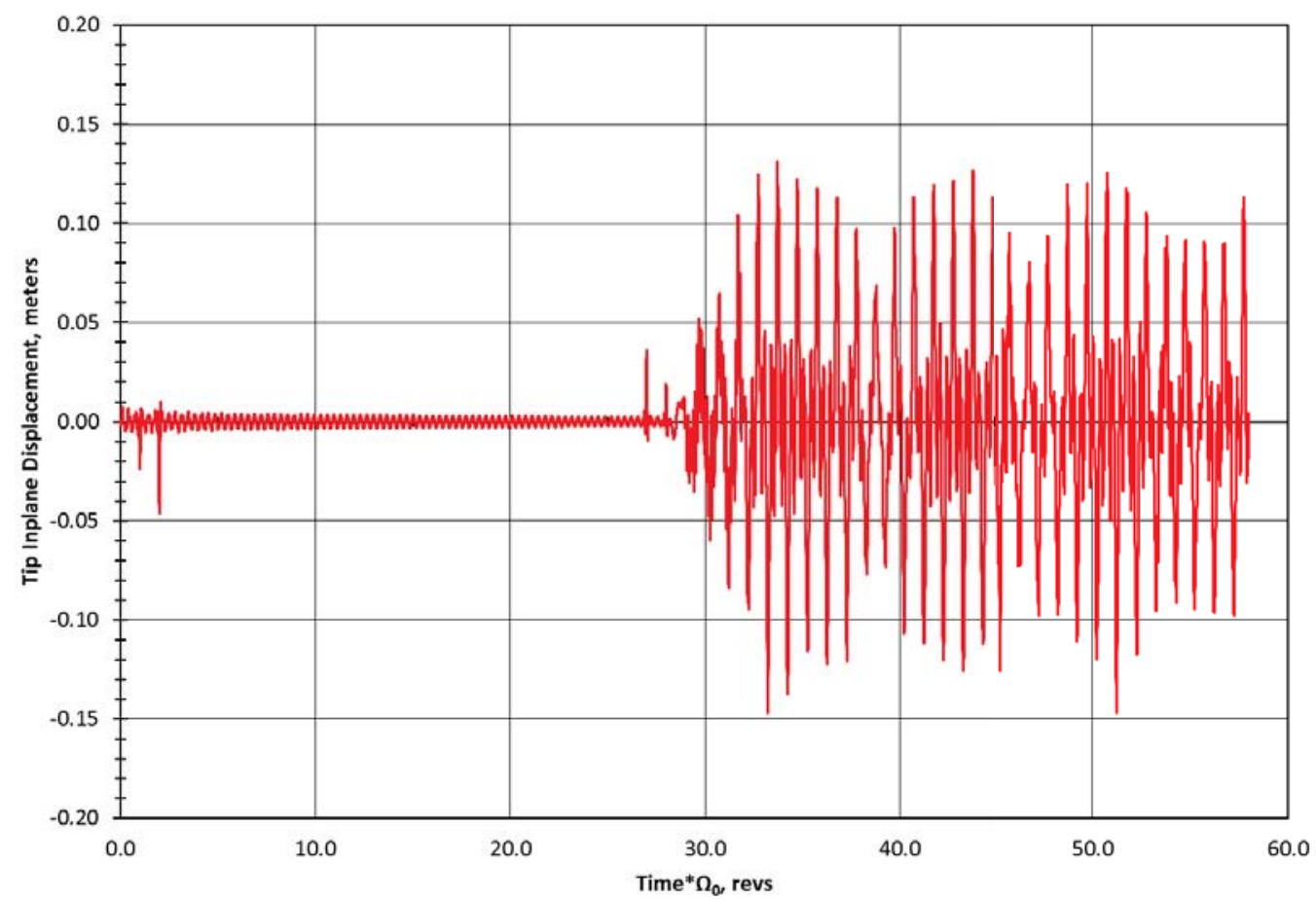

Figure 14. Case 2, 25 degree 1P cyclic pitch input: Blade tip in-plane displacement. 


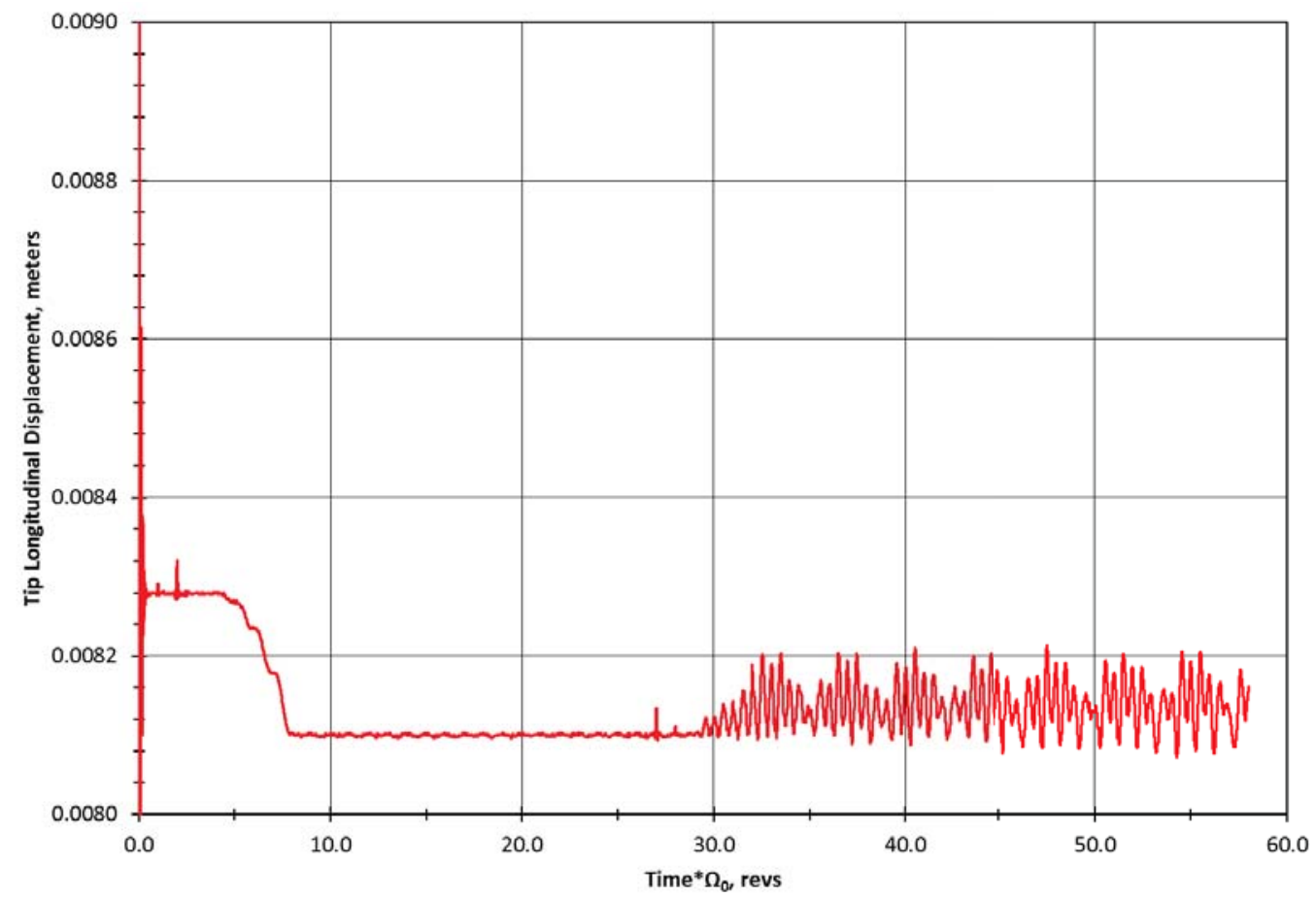

Figure 15. Case 2, 25 degree 1P cyclic pitch input: Blade tip axial displacement.

A comprehensive system identification of the heliogyro blade was not performed, however Fast Fourier Transforms (FFT) of portions of the tip displacement and twist time histories from Case 1 were used to estimate the flap, twist, and inplane blade natural frequencies. The frequencies from the FFTs are compared to those presented by MacNeal in Ref. 8 and repeated here in Table 2. For FFT analysis segments of the Abaqus time histories were chosen to identify the dominant flap, twist, and in-pane motion caused by the application of the solar radiation pressure load. Both twist and flap modal frequencies from the FFT estimates compare well with those predicted by MacNeal. However, for in-plane, the FFT estimate lies between the first and second frequencies from MacNeal, but is not close to either one. In Ref. 8, MacNeal mentions that he neglected the inplane elastic bending portion of the blade stiffness when solving for the inplane frequencies because the contribution was small for his heliogyro configuration. However, this assumption is not necessarily true for the HELIOS heliogyro blade. A handbook solution for a pinned-free beam frequency from Ref. 10, which accounts for the inplane bending stiffness yields a frequency of $3.174 \mathrm{P}$, adjusted to account for centrifugal stiffening due to the axial load. Since the tensile load in the heliogyro blade varies along the length, an average value was used to calculate this solution. Note that the handbook natural frequency is closer to the FFT value which indicates that the inplane elastic bending stiffness might not be negligible for the HELIOS heliogyro blade. Based on these results more work is needed to better understand the inplane natural frequencies of the HELIOS heliogyro blade.

\section{B. Heliogyro Solarelastic Stability Analysis}

Heliogyro dynamics and solarelastic ${ }^{12}$ stability studies are possible using the fully-coupled, finite element analysis methods described above, although these simulations are computationally expensive. More efficient heliogyro analytical tools appropriate for rapid design and trade studies are needed. Our approach, ${ }^{13}$ previously explored by Natori, ${ }^{14}$ adapts rotating beam equations of motion developed for conventional helicopter rotor blade analysis for use with the heliogyro. Complete details on the equations of motion used here, and their solution, may be found in Ref. 15. These equations can be numerically solved using MATLAB-based codes, and although not applicable to all heliogyro flight conditions, can be used to evaluate heliogyro structural dynamic and stability behavior for many restricted, yet still relevant, cases. One such case is for a heliogyro operating at zero blade pitch angle with the spin axis pointed toward the sun. For this case, the equations of motion can be transformed into a second-order set of ordinary differential equations with constant coefficients valid for small deflections. The eigenvalues of this system can be evaluated as a function of fixed rotational speed and incident solar radiation 
Table 2. Comparison of analytical heliogyro blade frequencies from MacNeal ${ }^{8}$ and FFT estimates from the coupled finite element analysis.

\begin{tabular}{cc||cc||cc}
\hline $\begin{array}{c}\text { MacNeal } \\
\text { Flap } \\
(\omega / \Omega)\end{array}$ & $\begin{array}{c}\text { FFT } \\
\text { Estimate } \\
\text { Flap } \\
(\omega / \Omega)\end{array}$ & $\begin{array}{c}\text { MacNeal } \\
\text { Twist } \\
(\omega / \Omega)\end{array}$ & $\begin{array}{c}\text { FFT } \\
\text { Estimate } \\
\text { Twist } \\
(\omega / \Omega)\end{array}$ & $\begin{array}{c}\text { MacNeal } \\
\text { Inplane } \\
(\omega / \Omega)\end{array}$ & $\begin{array}{c}\text { FFT } \\
\text { Estimate } \\
\text { Inplane } \\
(\omega / \Omega)\end{array}$ \\
\hline \hline 1.000 & 1.031 & 1.414 & 1.383 & 2.236 & 2.956 \\
2.449 & 2.438 & 2.646 & 2.555 & 3.742 & - \\
3.873 & 3.891 & 4.000 & 3.961 & 5.099 & - \\
5.291 & 5.297 & 5.386 & 5.32 & - & - \\
\hline
\end{tabular}

pressure to determine operational stability boundaries for a given heliogyro design. Example stability behavior trends for the HELIOS uniform reference blade are shown in Fig. 16.

Time history results show that the blade response is predictable and stable for two typical heliogyro root pitch profiles. The $\pm 25^{\circ}$ cyclic root pitch profile causes large amplitude tip twist oscillations but the response is still stable. The analyses included a small amount of material damping but the effects of the damping are not discernable in the time history results. This is consistent with earlier work by MacNeal ${ }^{8}$ and Blomquist ${ }^{11}$ that emphasizes the need for some means of controlling blade motion.

Here the imaginary parts of the blade eigenvalues, corresponding to the normalized, i.e., per revolution, blade modal frequencies, are tracked as a function of effective solar radiation pressure. A blade flutter instability, signified by coalescence of the fundamental in-plane and torsional vibration modes, is indicated near an effective solar radiation pressure of $4.1 \times 10^{-5} \mathrm{~Pa}$. This corresponds to a heliocentric distance of about $0.5 \mathrm{AU}$. Blade dynamics in the vicinity of $1.0 \mathrm{AU}\left(\sim 0.91 \times 10^{-5} \mathrm{~Pa}\right)$ are stable; at least for the nominal HELIOS rotational speed of 1 RPM. Further instabilities at higher solar radiation pressures are also indicated, most notably, a divergence instability near $5.3 \times 10^{-5} \mathrm{~Pa}$ and a higher frequency flutter condition at $5.7 \times 10^{-5} \mathrm{~Pa}$, although such post-critical instabilities are not physically significant.

Stability bounds for a given heliogyro blade design may also be mapped as function of rotor speed and effective solar radiation pressure. The stability boundary for the uniform HELIOS blade is shown in Fig. 17. Here, all unstable eigenvalues over the rotor speed range indicated have been tracked with the critical radiation pressure required to induce instability plotted against rotor speed. The lower bound in radiation pressure is indicated by the thick red line, with stable regions of rotational speed and radiation pressure lying below the line. Examination of this stability boundary plot indicates that at $1.0 \mathrm{AU}$ solar radiation pressures $\left(0.91 \times 10^{-5} \mathrm{~Pa}\right)$ the HELIOS heliogyrp blade will encounter a solarelastic instability when rotational speed drops below approximately 0.6 RPM.

Experimental verification of solarelastic stability characteristics would actually be an important goal of a heliogyro flight technology demonstration mission. ${ }^{2}$ In practice, this could be accomplished by gradually reducing heliogyro rotation speed through the application of blade collective pitch; pausing periodically to experimentally evaluate the sub-critical changes in blade modal frequencies. As the dynamics of the heliogyro are relatively slow in real-time, stability boundaries may be approached slowly, and backed away from relatively quickly by re-application of blade collective pitch to increase the vehicle spin rate.

American Institute of Aeronautics and Astronautics 


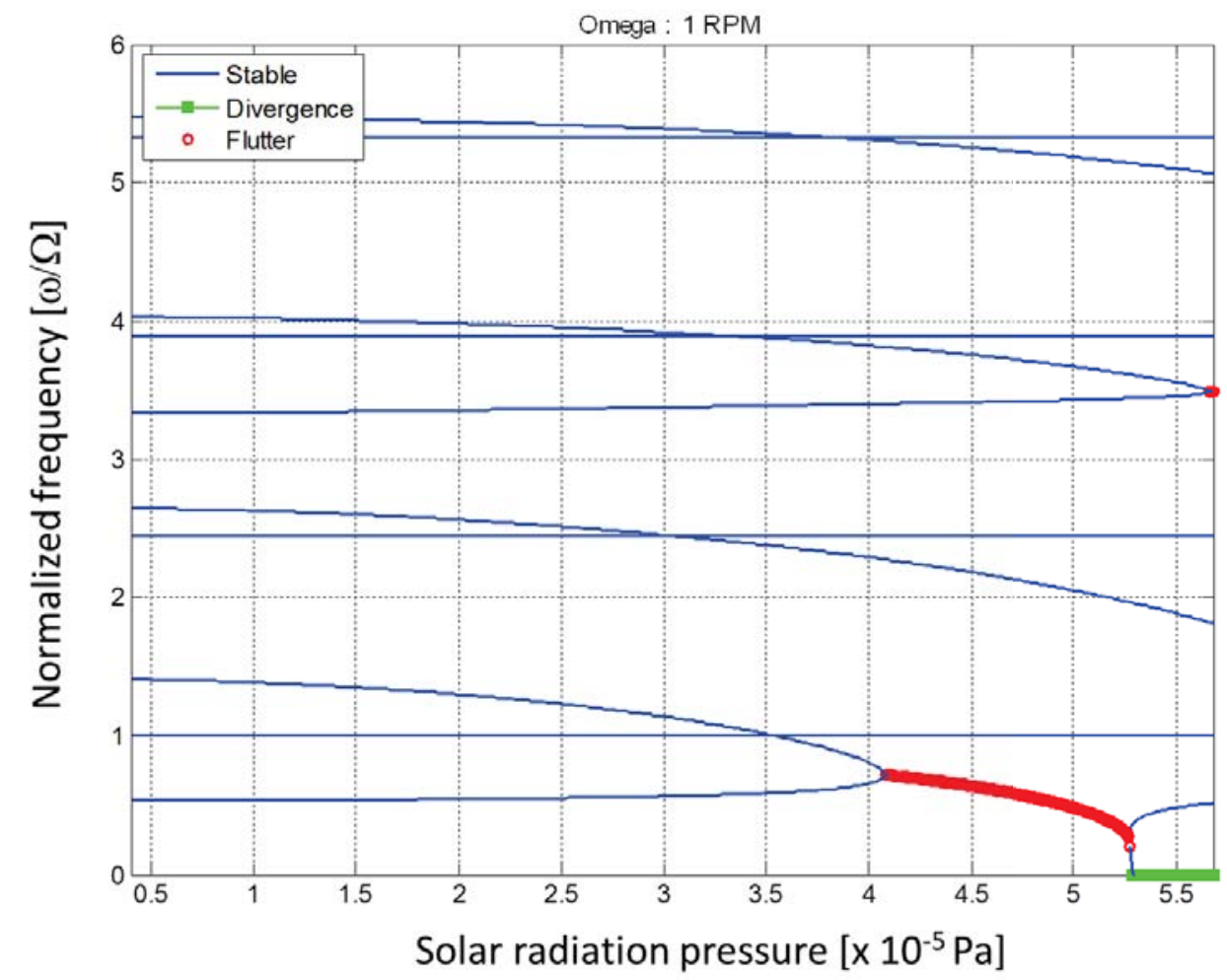

Figure 16. Eigenvalue stability analysis of the HELIOS uniform reference blade at the nominal rotational speed, $\Omega=1$ RPM, and zero sun angle.

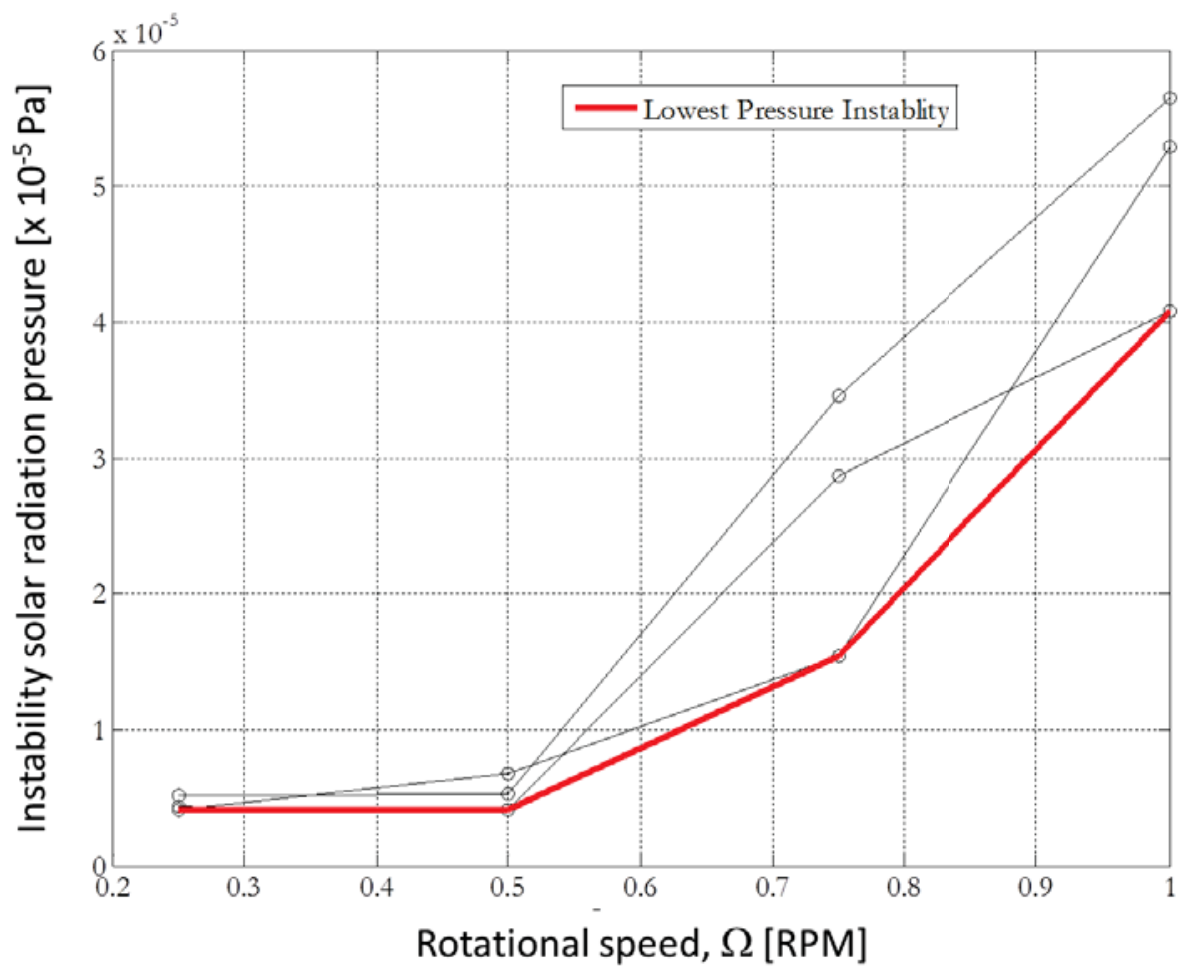

Figure 17. Solarelastic instability boundary of the HELIOS uniform reference blade. Zero collective pitch and sun angle are assumed. 


\section{Heliogyro Blade Stability During Deployment}

Solarelastic stability of the HELIOS heliogyro during deployment has also been studied. Figure 18 shows the instability radiation pressure and instability frequency of the HELIOS blades during deployment. The instantaneous stability of the blades may be examined at any stage of deployment, between the nominal initial deployment state (20 $\mathrm{m}, 6 \mathrm{RPM}$ ) and the final fully deployed configuration (220 m, 1 RPM). The stability boundary map shown here is calculated for the sun directly overhead, i.e., on the spin axis. An interesting behavior occurs when the system transitions from a divergence instability, the lowest radiation pressure instability at the initial deployment state, to a flutter instability, the lowest radiation pressure instability for the fully deployed configuration. At the transition from divergence to flutter, the radiation pressure required to cause an instability drops significantly. This is a concern as HELIOS blade deployment, which would occur at 1.0 AU solar radiation pressures, will likely have to traverse this transition region.

To confirm the phenomenon, we compared our analytical results to fully coupled HELIOS heliogyro blade simulations using our Abaqus nonlinear finite element model, described previously. For the comparison, we selected an intermediate deployed blade span of $90 \mathrm{~m}$, and examined stability for spin rates between 1 RPM and 2.2 RPM. In Fig. 19 the stability boundary for different spin rates as predicted by the analytical model is shown by the solid black line. A large decrease in the instability radiation pressure is seen near a rotational speed of 1.4 RPM, which correlates with the transition from a divergence instability to a flutter instability.

To validate these predictions we conducted Abaqus simulations using solar radiation pressure conditions of both $0.1 \times 10^{-5} \mathrm{~Pa}$ and $0.9 \times 10^{-5} \mathrm{~Pa}$ for different spin rates. Solutions that remained bounded after 50 simulated revolutions were deemed stable. Figure 19 identifies configurations with stable Abaqus simulations using a green dot, and unstable configurations using a red dot. As seen in the figure, stable and unstable Abaqus simulations appear to lie within the regions predicted by the analytical model.

It is important to note that for the simulations shown in Fig. 19, the Abaqus model assumes the hub of the spacecraft is rotating at a fixed, prescribed rate, which is consistent with the blade dynamics theory used in the analytical model. Interestingly, when the Abaqus model is not hub driven, i.e., when the central spacecraft bus is free to rotate in response to torques generated by solar radiation pressure acting on the blades, the simulations are stable for all spin rates and radiation pressure conditions examined in Fig. 19. This free-spinning hub representation is more representative of the actual HELIOS flight configuration, and while the phenomenon predicted by our fixed-spin-rate hub analytical model is validated, we do not anticipate that the actual HELIOS solar sail, with a central bus free to rotate in response to forces acting on the blades, will encounter the narrow transition instability region predicted by the driven-hub models while deploying.

\section{Nonlinear Heliogyro Blade Control Investigations}

The heliogyro blade is so flexible that normal operations excite the blade very near the first structural twist mode, and settling time goals preclude traditional controller gain stabilization. Furthermore, spacecraft mass and acceleration goals preclude the addition of significant structural stiffening. Past work on blade twist stability has been limited to the linearized dynamics, ${ }^{5,8,11,16,17}$ but nonlinear effects can significantly alter the torsional dynamics, even at moderate excitation amplitudes. For example, Fig. 20 shows a simulation using the twist-only, nonlinear, finite element "membrane ladder" model (FEM) of Ref. 18. Shown are blade root and tip torsional responses for (a) $25^{\circ}$ and (b) $35^{\circ}$ once-per-rev (1P) cyclic excitations, along with the reference (i.e. desired) root signal. Although not all higher harmonics are completely damped, tip oscillations are reasonably bounded in the $25^{\circ}$ case. By contrast, the response to a $35^{\circ}$ cyclic root pitch command is unacceptably large with the blade twisting completely around.

American Institute of Aeronautics and Astronautics 

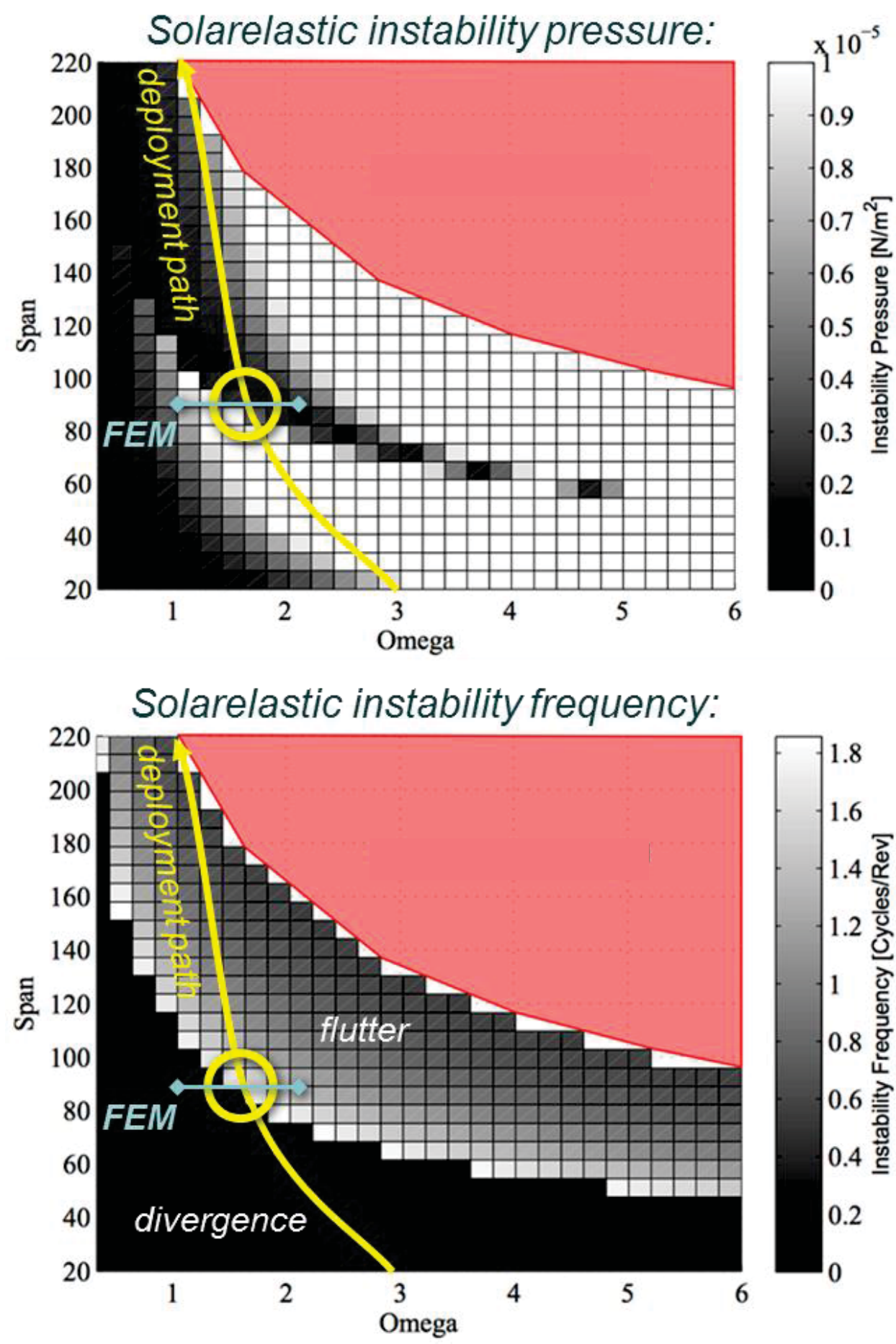

Figure 18. Solarelastic instability pressure (top) and frequency (bottom) as a function of deployed blade radius (span in $\mathbf{m}$ ) and rotational speed (RPM). The approximate span-versus-spin-rate deployment path is indicated in yellow. Pink regions were not included in the analysis domain. 


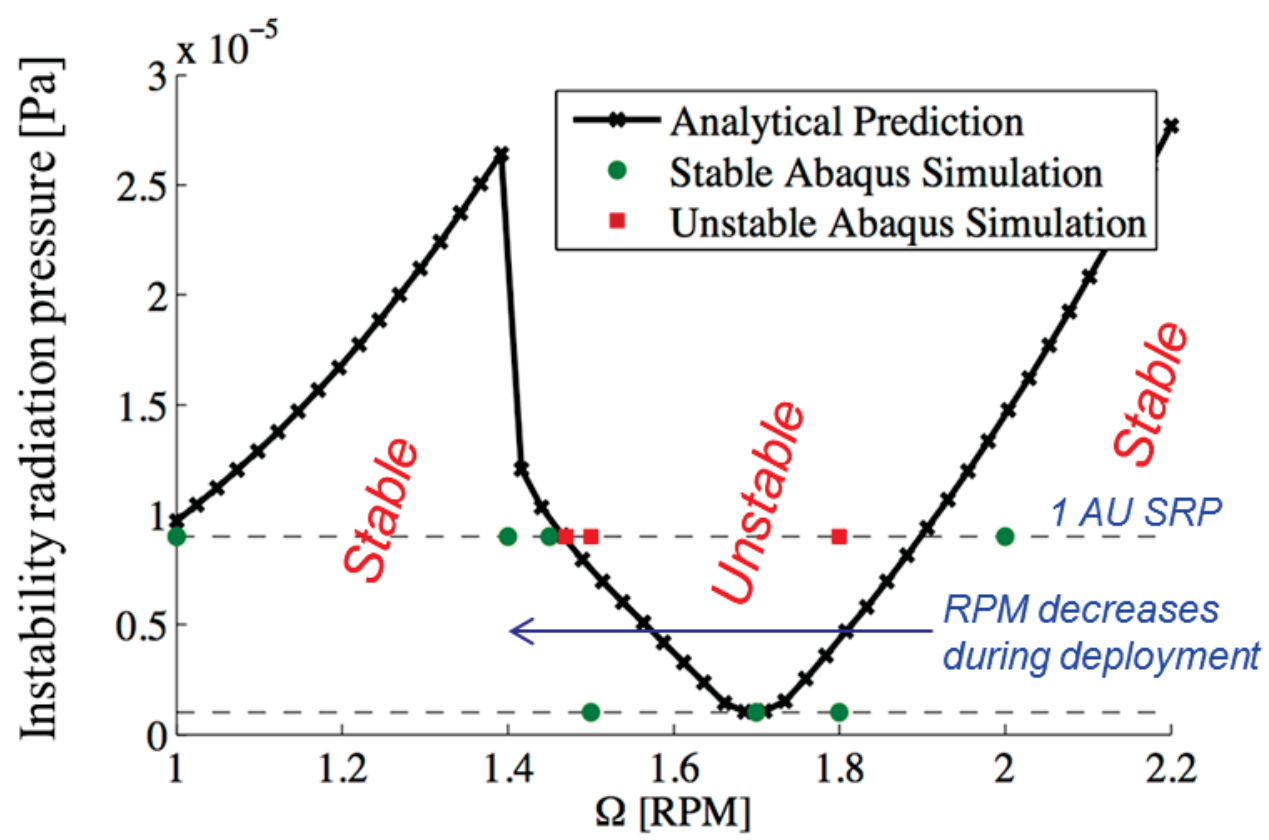

Figure 19. Comparison of analytical and Abaqus FEM solarelastic divergence-to-flutter instability behavior.

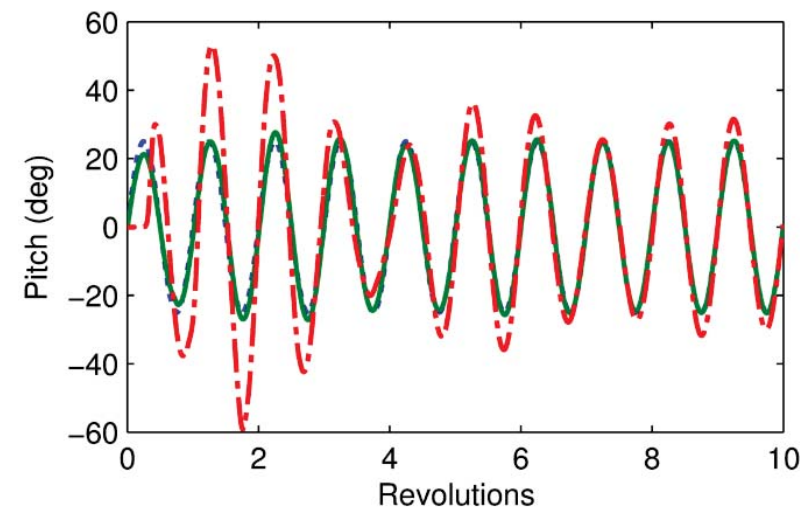

a) $25^{\circ}$ cyclic amplitude

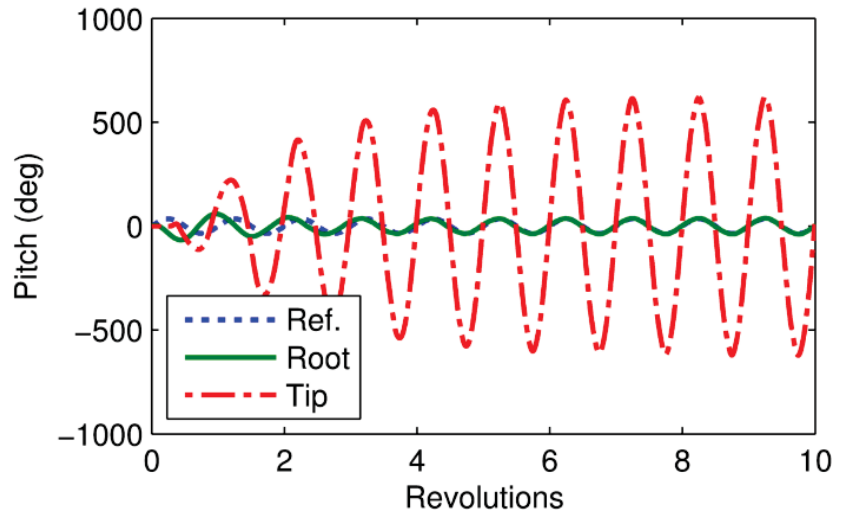

b) $35^{\circ}$ cyclic amplitude

Figure 20. Dynamic simulations of membrane ladder FEM for 25 and 35 degree cyclic pitch inputs.

The cause of this undesirable response is the first torsional mode frequency shifting below the 1P cyclic frequency due to amplitude-dependent nonlinearities. One way to investigate this phenomenon is via a solution to the blade's steady-state response using a single-frequency Fourier expansion at the excitation frequency. ${ }^{18}$ Figure 21 illustrates this steady-state solution with a two-dimensional, nonlinear describing function (DF), analogous to the linear system's frequency response function. It shows the blade root-to-tip amplitude response vs. cyclic pitch amplitude and frequency with the half-P and 1P cyclic curves overlaid (black lines). The first structural mode from the linear case (drawn at a $1=0^{\circ}$ ) initially shifts down in frequency as the root amplitude increases, but the system retains a quasilinear frequency response. At around $30^{\circ}$, a bifurcation occurs, and the system behavior becomes distinctly nonlinear. The responses shown in Fig.21 are on either side of this shifting first mode.

American Institute of Aeronautics and Astronautics 


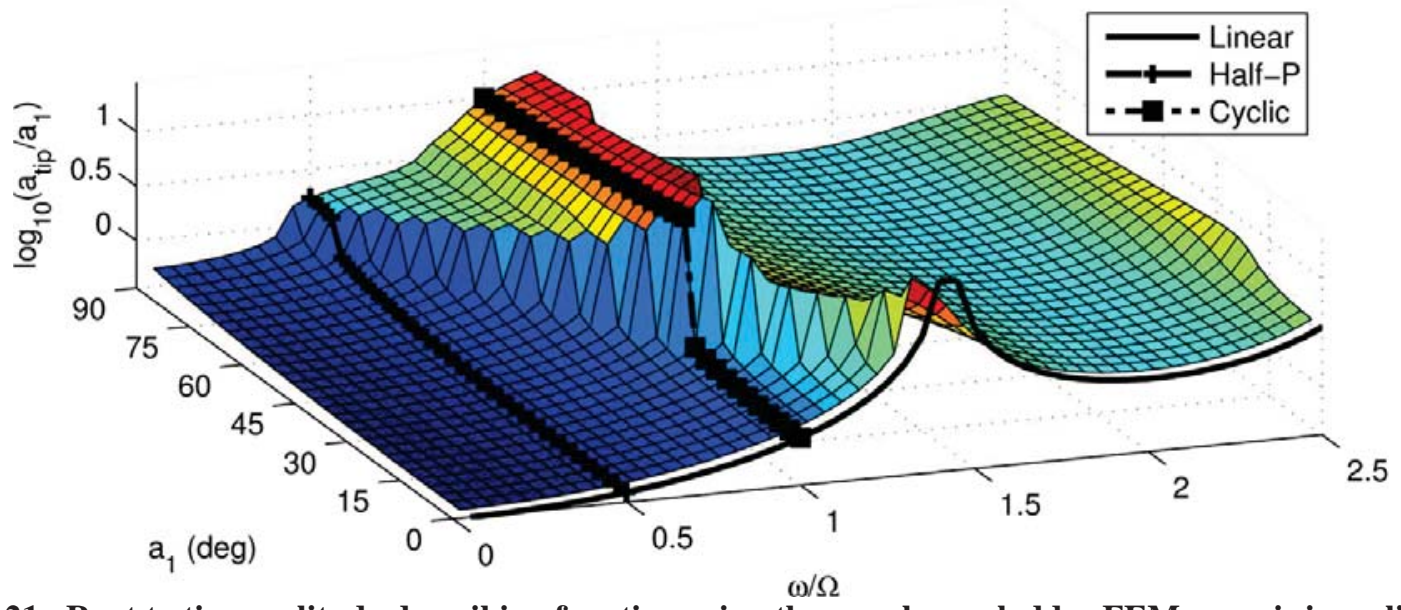

Figure 21. Root to tip amplitude describing function using the membrane ladder FEM: $x$-axis is cyclic pitch frequency in cycles/rev, y-axis is blade root cyclic pitch amplitude, z-axis is root-to-tip response magnitude.

One approach for mitigating undesirable shifts in torsion mode frequencies is to increase the centrifugal stiffening of the blade. This may be accomplished using a larger tip mass, although this additional mass should be concentrated at the twist axis rather than distributed across the blade chord to minimize increases in the tip rotational moment of inertia. A second method of increasing centrifugal stiffness is to redirect the spanwise tension acting along the blade to the leading and trailing edges. In practice, this load distribution could be accomplished with tension-carrying elements at the edges of the blade (e.g., high tensile strength cables or tapes) with periodic spanwise attachment of membrane sail panels to the edge members. The resulting blade structural dynamics would more closely resemble those of a spinning rope ladder than a uniform membrane sheet. This approach was ultimately adopted for the $15 \mathrm{~km}$ diameter JPL heliogyro studied in the 1970s. ${ }^{2}$ Figure 22 shows a simulation of the rope ladder blade using both edge stiffeners and additional tip mass, each of which add about $7 \%$ to the blade mass. Response to a $40^{\circ}$ cyclic pitch command is now shown to be stable, with blade flatness and settling time greatly improved. This initial work suggests that non-linear effects of torsion mode frequency shifts can be avoided and reasonable blade flatness maintained with modest structural design changes.

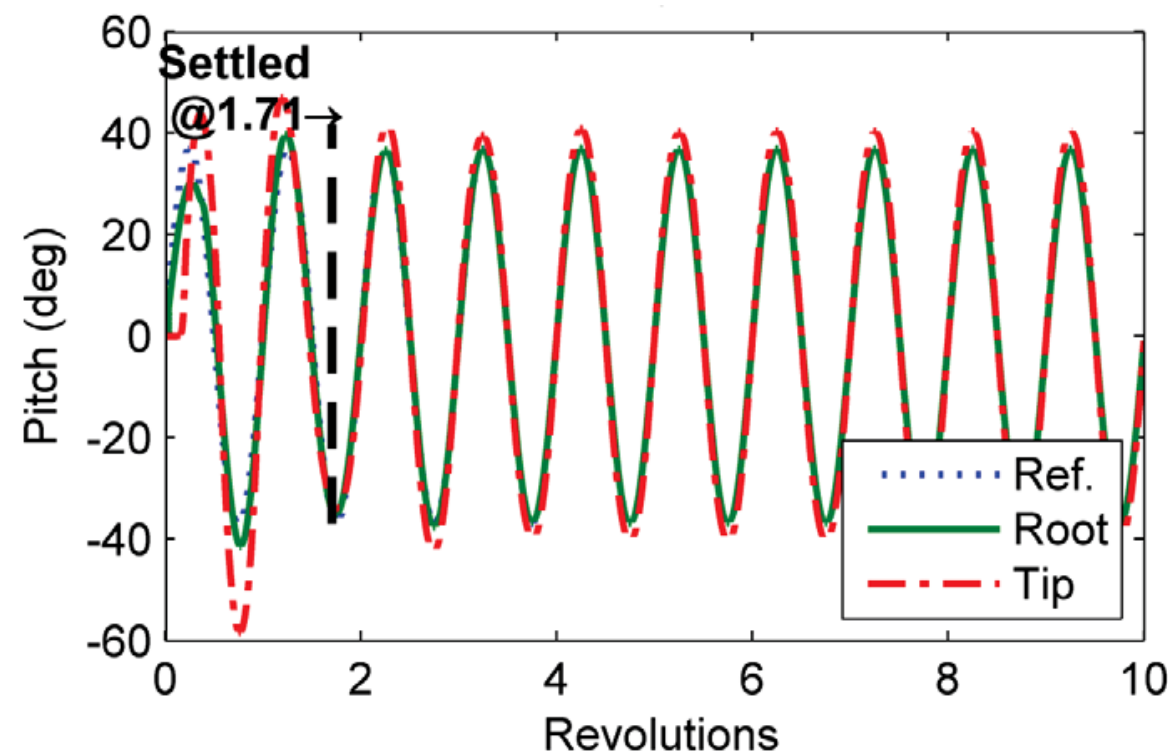

Figure 22. Dynamic simulation of rope ladder FEM with edge reinforcing, tip point mass, and $40^{\circ}$ cyclic pitch inputs. 
The simulations above incorporate a proportional/derivative/feed-forward (PDFF) root pitch control law to damp the natural response, as there is no material damping in these models. Feed-forward greatly improves blade root controller tracking and settling time performance, but this requires knowledge of the blade's steady-state motion. An effective way to calculate the nonlinear (amplitude-dependent) feed-forward coefficients is by harmonic Fourier expansion of the blade's steady-state response, similar to the process used for Fig. 21 but incorporating additional harmonic frequencies. ${ }^{18}$ Fast Fourier transforms (FFT) of the blade dynamic response in simulation show that five harmonic frequencies $\{0,0.5,1,1.5,2$ cycles/rev $\}$ are sufficient to capture $99.5 \%$ of the steady-state response power spectrum. The resulting PDFF control law settles blade motion within 3 revs, or three minutes for the nominal 1 RPM HELIOS heliogyro spin rate. Assuming instantaneous changes to the blade pitch profile is therefore a reasonable simplification for preliminary attitude and trajectory analyses. Even in low Earth orbit, where the orbit period is a relatively short 100 minutes, the heliogyro could vary the pitch profile effectively several times per orbit.

Incorporating these improvements, the flat-blade assumption is generally reasonable for preliminary attitude and trajectory analyses, but higher harmonics arising naturally can either increase or decrease propulsion and attitude control performance by as much as $25 \% .{ }^{18}$ The PDFF controller used here effectively damps higher harmonics, but only at the blade root. Future investigation will examine whether control of the higher harmonics can improve performance over the nominal flat blade.

\section{E. Heliogyro Blade Dynamics and Control Testbed}

Although testing full-scale spinning heliogyro blades is impossible on the ground, some useful blade dynamics and control experiments can be performed in a 1-g laboratory environment. In particular, experiments on short sections of heliogyro blades suspended under 1-g can provide both insight into the dynamics of membrane or membrane-like structures and guide the development of flight heliogyro blade control and metrology systems. A simple heliogyro blade dynamics and control testbed system is shown in Fig. 23. This system consists of surrogate full-scale, $7 \mathrm{~m}$ x $0.75 \mathrm{~m}$ single-bay heliogyro blade suspended vertically, a root pitch actuator, and a single video camera, also located near the blade root. The blade bay surrogate consists of carbon battens and stainless steel surgical wire edge tendons, and resembles a rope ladder. This rope ladder surrogate mitigates much of the influence of air currents and minimizes unwanted aerodynamic damping. The motion of the bay was measured, at eight large circles aligned with the rungs, by using the videogrammetry system. The root actuation and the videogrammetry system were both controlled by a LabVIEW program that was also used to analyze video data.

A simplified Matlab analytical model of the four-batten rope-ladder was also developed to support blade control design studies. The model assumes that blade motion is characterized by pitch motion of the battens,i.e. no flap or vertical motion, and that the edge tendons have negligible bending and torsion stiffness and are inextensible. The masses of the battens were adjusted to calibrate the analytical model in both frequency and mode shape to the test results. The analytical frequencies for the 1 st and $2^{\text {nd }}$ modes were $0.19 \mathrm{~Hz}$ and $0.59 \mathrm{~Hz}$ respectively, with greater than $99 \%$ coherence with the experimentally measured frequencies.

Open-loop response of the surrogate blade to a $60^{\circ}$ step input is shown in Fig. 24. Negligible damping is observed, which is consistent with expected behavior of an actual flight heliogyro blade. Response to a smoothed ramp 60 degree root pitch input is shown in Figure 25. The difference in the responses for the four battens is insignificant. A simple proportional-integral-derivative (PID) feedback controller was also added to the smoothed ramp input. Various control weighting schemes using the batten pitch angles were investigated to optimize settling time, although using the pitch response from the most inboard batten only proved to be the most effective. The closed loop response of the PID controller with the ramp-sinusoid is shown in Fig. 26. These preliminary blade control experiments have been encouraging and suggest that profiled pitch commands or relatively simple feedback control schemes can control the heliogyro blade torsional response. In the future, more realistic ground experiments will be conducted, including in vacuo 1-g blade control studies on actual membrane blade bay segments. These experiments will also be performed with increasingly more flight-like actuator and camera hardware and control software.

\section{F. In Vacuo Heliogyro Blade Spin Dynamics Experiments}

Although exact dynamic testing of full-scale spinning heliogyros is not possible on the ground due to the presence of gravity, experiments on small sub-scale spinning membrane systems can be performed under one-g. The results from these experiments can be used for validating heliogyro structural dynamics models and for building confidence in analytical simulations of the full-scale vehicle. Such experiments were proposed during the JPL heliogyro study of the 1970s, although no experiments were performed. ${ }^{20}$ Some earlier heliogyro spin dynamics validation experiments were attempted in air by MacNeal, but were severely complicated by atmospheric effects. ${ }^{8}$ As an inexpensive pathfinder experiment, we have recently begun testing small-scale spinning membrane blades in

American Institute of Aeronautics and Astronautics 


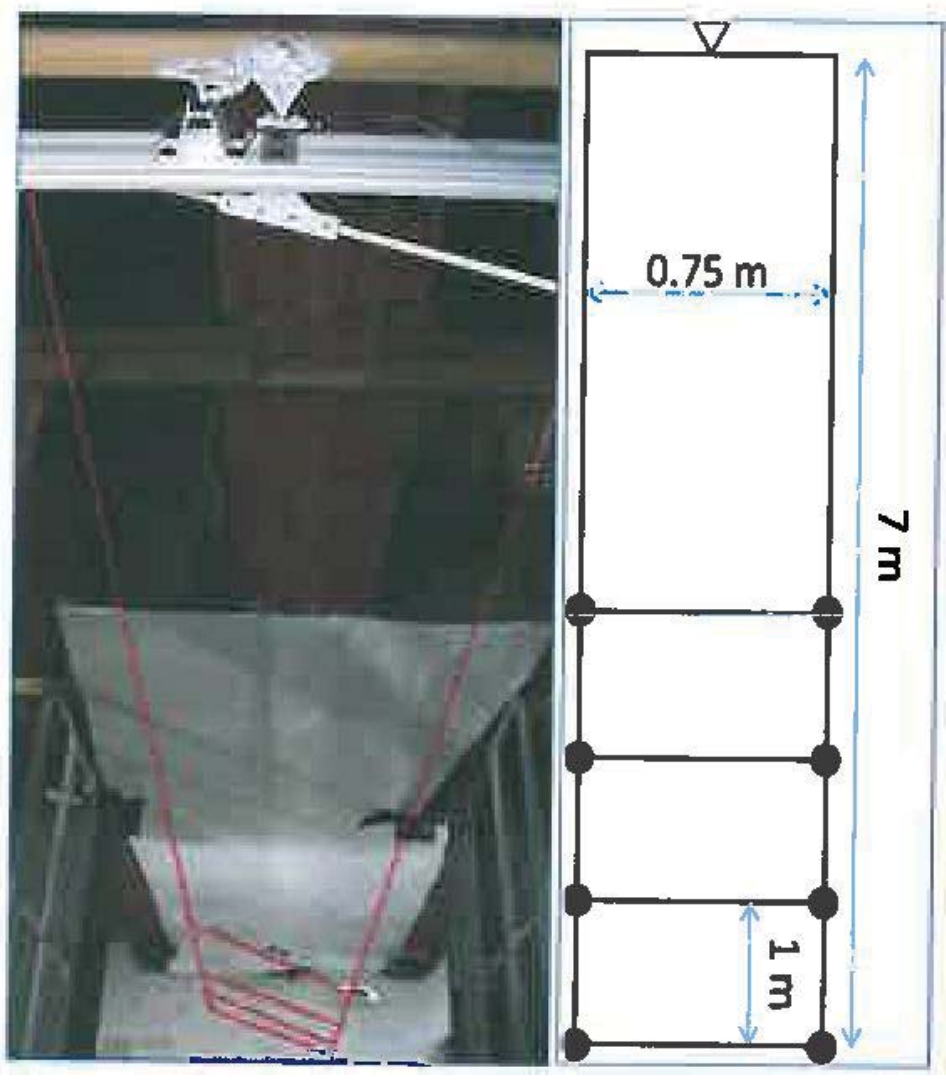

Figure 23. Full-scale surrogate model heliogyro blade bay with root actuator.

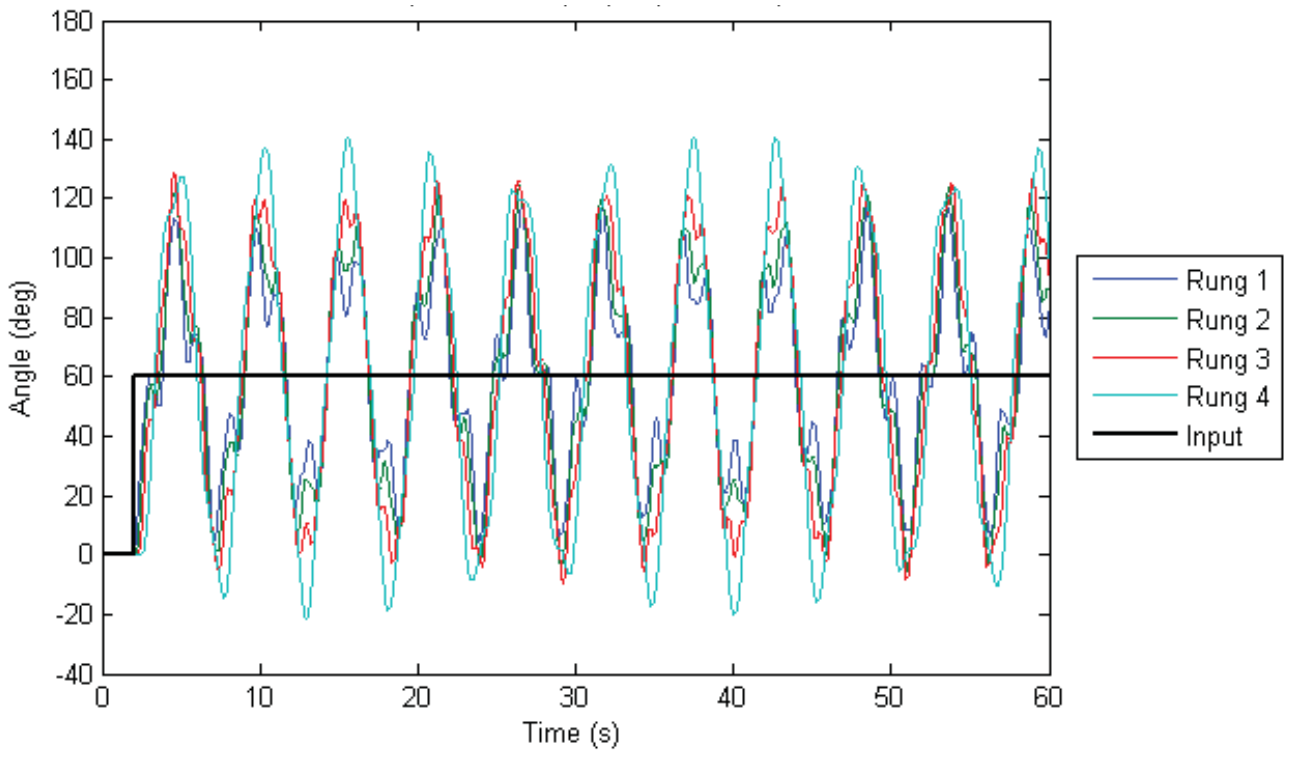

Figure 24. Pitch angles for root actuation and batten responses for a $60^{\circ}$ step input with no control. 


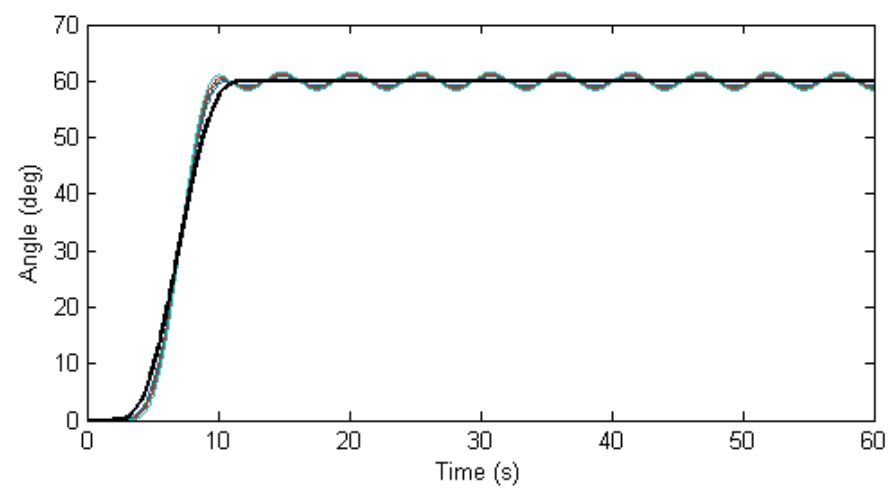

Figure 25. Pitch angles for root actuation and batten responses for a smoothed ramp pitch input.

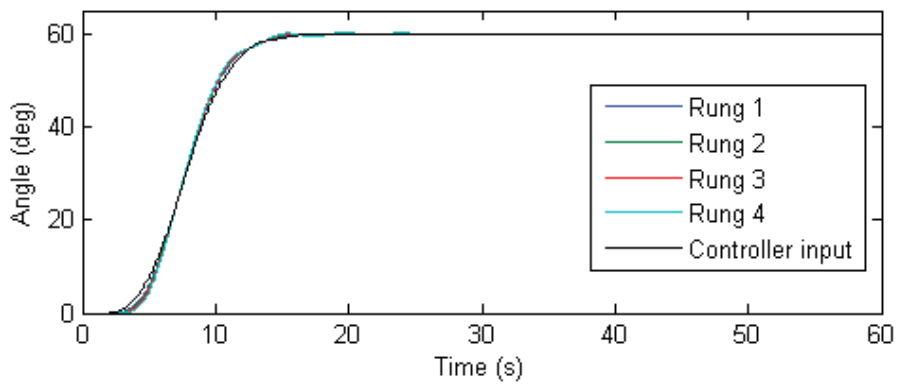

Figure 26. Pitch angles for root actuation and batten responses for a smoothed ramp input with PID control.

our 2.5-m diameter vacuum sphere using a system constructed around low-cost, commercially available radiocontrolled helicopter components.

1. In Vacuo Spin Dynamics Experiment Apparatus

Figure 30 shows the test apparatus in $2.5 \mathrm{~m}$ diameter vacuum sphere. Also shown is an expanded view of the rope ladder blade used for testing. In this test, the Kapton membrane blades, initially reported in Refs. 4 and 21, were replaced with a rope-ladder blade model $0.46 \mathrm{~m}$ long by $0.048 \mathrm{~m}$ wide to reduce low pressure air effects during testing. These effects were still apparent during earlier tests of membrane blade models at the rough vacuums ( $<1$ Torr) achievable in the chamber. To improve the camera field-of-view, camera pedestals were raised to $0.1 \mathrm{~m}$ from the blade root spin plane. Video data, collected in the rotating frame of reference, was processed using Labview to recover two dimensional displacements of the targets on the blade. A total of 1.0833 seconds of 30 frame-per-second video data was used for identification of the blade dynamics. The nominal spin rate during blade video data collection was 110 RPM $(\Omega=1.833 \mathrm{~Hz})$. Video data was corrected using the line-of-sight angle as a correction factor for target motion toward and away from the single hub-mounted camera used here. Targets closest to the camera had the largest angle; about 25 degrees. The correction angle was set to 0 degrees at the tip. For purposes of system identification, drive system vibration was sufficient to excite most blade dynamics. Input disturbance information was not used in the system identification approach adopted here, although, in general, this can create difficulties when attempting to identify dynamics if the disturbances forces do not excite the vibration modes of interest.

2. Rope-Ladder Finite Element Model Development

An Abaqus finite element model (FEM) of the rope-ladder test article shown in Fig. 27 was created in order to generate predictions of natural frequencies and mode shapes for comparison with with the experimental system identification results. The finite element model consists of five longitudinal tendons and five chordwise battens, similar to the actual rope ladder blade model shown in Fig. 27. The longitudinal tendons were modeled using stiff T3D2 truss elements that are intended to behave like rigid links between the battens. The density of the truss elements was chosen to produce the same mass as the actual longitudinal tendons. The battens were modeled using stiff B31 beam elements that are intended to behave like rigid bars. The density for each batten in the FEM was chosen to match the mass of the corresponding actual batten. Multiple beam elements per batten were used to provide a more accurate mass moment of inertia about the longitudinal axis of the test article. Point masses were placed at the leading and trailing edge nodes on the battens. The point masses include mass from the videogrammetry targets and Kapton tape.

American Institute of Aeronautics and Astronautics 


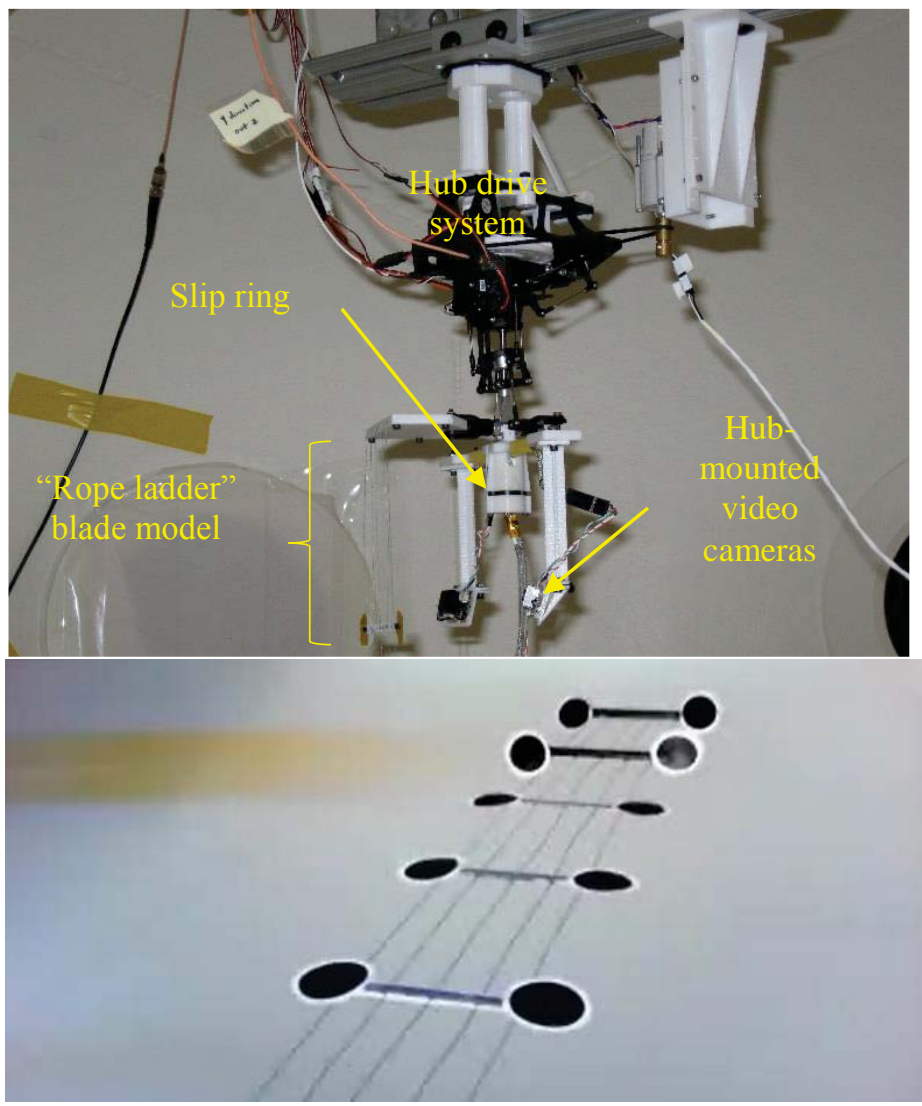

Figure 27. (Top) Heliogyro blade spin dynamics testbed in vacuum chamber. (Bottom) Hub-mounted camnera view of spinning rope-ladder blade with videogrammetry tracking targets.

The Abaqus analysis of the rope ladder included four solution steps to generate the reduced dynamic model. Nonlinear geometric effects were included in the analysis so that the preloaded configurations would convey to subsequent solution steps. The rope ladder FEM was fixed in all directions at the spin axis to simulate blade deformation relative to a rotating coordinate system. The first static solution step included the Abaqus static equivalent centrifugal load (CENTRIF) to stiffen the blade similar to the actual spinning test article. The second solution step included a 1-g gravity load parallel to the spin axis. The third solution step generates the natural frequencies and mode shapes for the preloaded model, and the fourth solution step uses those mode shapes to generate a reduced dynamic model. The reduced dynamic model included the first 20 modes as generalized degrees-of-freedom. Displacements were recovered for all degrees-of-freedom at the 10 target locations and two nodes near the root of the rope ladder. Natural frequencies and mode shapes from the reduced dynamic model are presented below.

\section{Output-Only System Identification of a Spinning Heliogyro Blade}

System identification of the spinning heliogyro blade presented some unique identification challenges because the test was conducted without measured disturbance inputs. Consequently, the blade responses observed during test were primarily the result of once-per-revolution forces acting at the center hub due to system imbalances. Because the test was conducted at a constant speed, these unmeasured centrifugal forces are periodic with a frequency of 1.0833 $\mathrm{Hz}$. This periodicity in the disturbance forces can be used to identify the vibration modes and frequencies of the system, provided that the disturbances are rich enough to excite the vibration modes of interest. Note that for purposes of system identification, any unknown periodic disturbance $u_{d}(k)$ can be written as:

$$
\left\{\begin{array}{c}
x_{d}(k+1) \\
u_{d}(k)
\end{array}\right\}=\left\{\begin{array}{c}
A_{d} \\
C_{d}
\end{array}\right\} x_{d}(k)(1)
$$

These disturbances act on the blades to produce a response $y(k)$ such that

American Institute of Aeronautics and Astronautics 


$$
\left\{\begin{array}{c}
x(k+1) \\
y(k)
\end{array}\right\}=\left[\begin{array}{ll}
A & B \\
C & D
\end{array}\right]\left\{\begin{array}{c}
x(k) \\
u_{d}(k)
\end{array}\right\}
$$

After combining Eqs. (2) and (3), the resulting system can be represented as

$$
\begin{gathered}
\left\{\begin{array}{c}
x(k+1) \\
x_{d}(k+1)
\end{array}\right\}=\left[\begin{array}{cc}
A & B C_{d} \\
0 & A_{d}
\end{array}\right]\left\{\begin{array}{c}
x(k) \\
x_{d}(k)
\end{array}\right\} \\
y(k)=\left[\begin{array}{ll}
C & D C_{d}
\end{array}\right]\left\{\begin{array}{c}
x(k) \\
x_{d}(k)
\end{array}\right\}
\end{gathered}
$$

Note that Eq. (4) implicitly includes the unknown external disturbance. Also note that in this form of the system equations, standard realization algorithms like those discussed in Ref. 21 can be applied to recover the system parameters $[A, B, C, D]$. However, from an implementation viewpoint, one difficulty with this approach is that the unknown periodic disturbance parameters $\left[A_{d}, C_{d}\right]$ are recovered along with the system parameters. Furthermore, parameters that appear as products, e.g., $B C_{d}$, cannot be easily separated into individual terms. Nonetheless, the system frequencies and mode shapes can be identified from the system parameters $[A, C]$. Results for heliogyro blade system identification using this process are shown below.

4. System Identification Results for the Spinning Sail Model

The identification process just described was implemented using time histories obtained from videogrammetry. In order to sort out system modes from other fictitious modes appearing in the identified system parameters in Eq. (4), a screening process was used whereby multiple realizations of the system parameters using the ERA algorithm in Ref. 21 were used to identify only the system modes. This process assumes that the system modes are consistently identified from multiple realizations of the system. In contrast, results for fictitious modes vary from realization to realization. Moreover, true system modes are characterized by their frequency, damping, and mode shapes. For example, in this problem the identification process used eight realized systems with a number of states ranging from 6 to 270 . These models were obtained by changing the assumed system order used during the realization process. From these eight systems, identified modes across multiple realizations were considered to be system modes if their frequencies were a) within $\pm 1 \mathrm{~Hz}$, b) had similar mode shapes with orthogonality better than 0.85 , and c) damping values less than $2 \%$. In other words, if a mode identified for the system of order 270 appeared multiple times, then the mode was classified as a system mode. A summary of the identification results for the spinning tests are shown in Table 3.

Frequency values in Table 3 are shown as a ratio of the identified frequencies divided by the rotational speed $\Omega=$ $1.0833 \mathrm{~Hz}$. Also shown within brackets is the variation in the identified frequency and damping values. To help visualize the main system modes, Fig. 28 illustrates the first four identified modes. Note that the first mode is combined bending/torsion mode; the second mode is an out of plane bending mode; the third mode is a torsion mode; and higher modes appear to have bending and torsion combined. By comparison, Fig. 29 shows the first four modes predicted using the FEM. In this case, the first mode is an in-plane mode, followed by an out-of-plane bending mode, torsion mode, and another out-of-plane bending mode. Note that the FEM modes show the blade root boundary nodes, whereas test results have those nodes removed.

To quantify similarities between the FEM model and test, Fig. 30 shows orthogonality values for five identified modes versus the FEM. Across the abscissa are the FEM normalized frequencies and the ordinate shows the normalized identified frequencies, as shown in Table 3. A black square corresponds to an orthogonality value of 1 , i.e., both experimental and analytical mode shapes are identical. From these results note that the second identified mode at 1.20 is similar to the second FEM model at 1.1, which is an out of plane bending mode. Also, the third mode identified at 1.58 is similar to the analysis mode at 1.24 . In both cases the orthogonality value is greater than 0.9 , based on data from nine targets in two directions.

Future modifications to the FEM and experimental apparatus should improve correlation. Modeling improvements will include the addition of mount and hub structural dynamics. Future spin dynamics experiments will be performed in our larger $2.5 \mathrm{~m} \mathrm{x} 5 \mathrm{~m}$ thermal vacuum chamber under higher vacuum conditions. This should minimize unsteady aerodynamic effects more fully, allowing us to better measure blade transient dynamics and damping behavior on both rope ladder and membrane heliogyro blade dynamics validation models.

American Institute of Aeronautics and Astronautics 


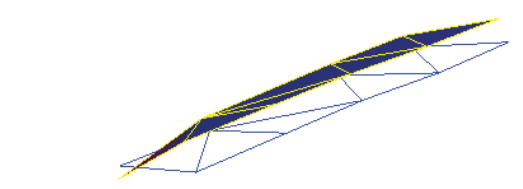

Mode 3 Freq $=1.58$
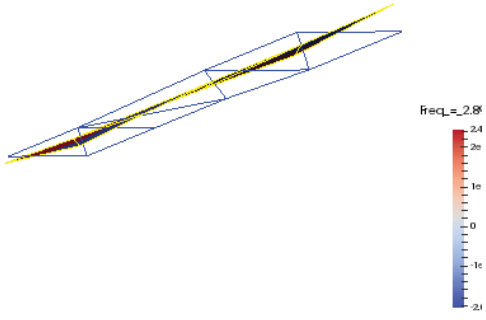

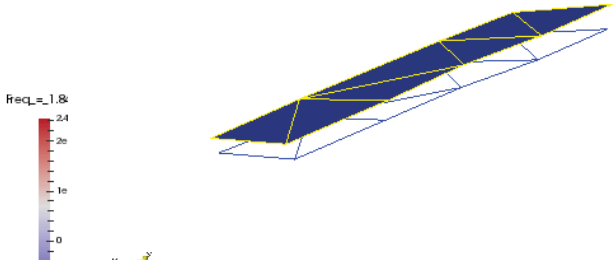

Mode 4 Freq $=4.01$
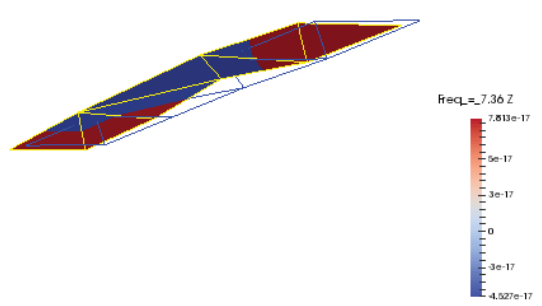

Figure 28. Experimentally identified modes using videogrammetry data.

Mode 1 Freq $=0.49$

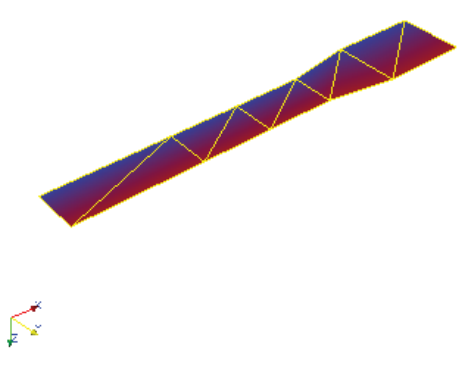

Mode 3 Freq $=1.24$
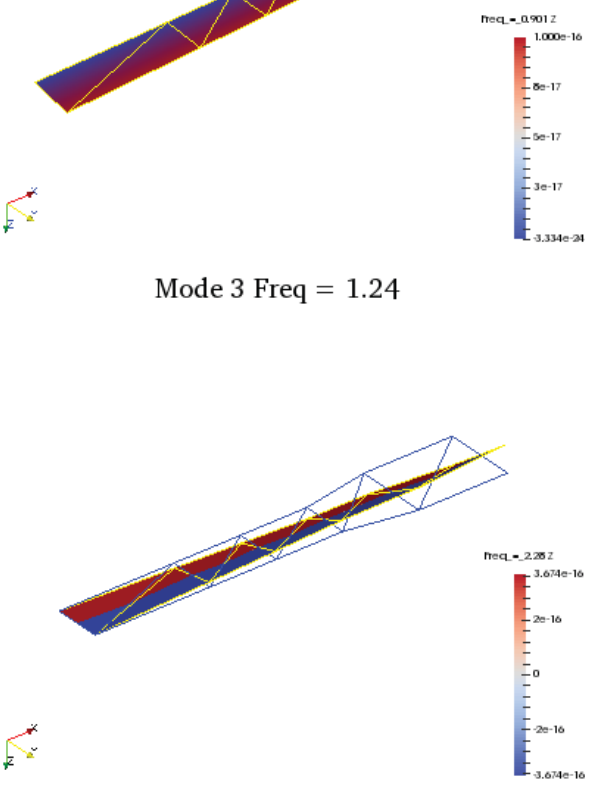

Mode 2 Freq $=1.10$
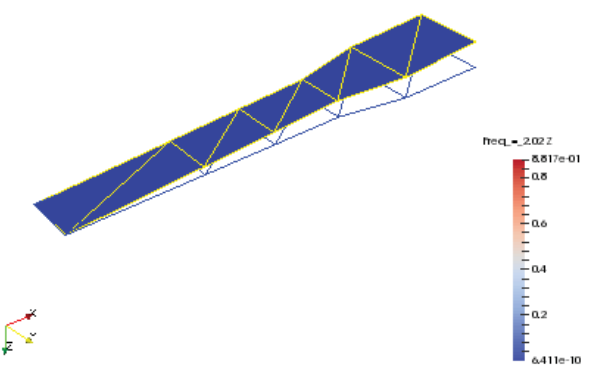

Mode 4 Freq $=2.37$

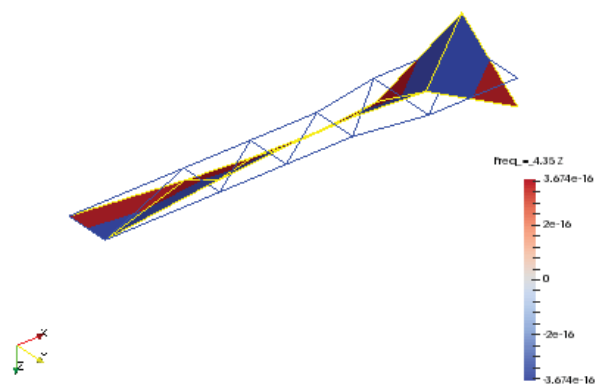

Figure 29 Predicted rope-ladder blade modes using the FEM model 
Table 3. Identified system modes and modal parameters for the spinning rope ladder blade

\begin{tabular}{ccccc} 
Mode & $\begin{array}{c}\text { Freq. } \\
(\omega / \Omega)\end{array}$ & $\begin{array}{c}\text { Freq. } \\
\text { Variation } \\
(\mathrm{Hz})\end{array}$ & $\begin{array}{c}\text { Damp } \\
(\%)\end{array}$ & $\begin{array}{c}\text { Damp } \\
\text { Variation } \\
(\%)\end{array}$ \\
\hline \hline 1 & 1.00 & {$[1.00,1.02]$} & 0.05 & {$[0.00,1.74]$} \\
2 & 1.20 & {$[1.20,1.21]$} & 0.39 & {$[0.00,0.40]$} \\
3 & 1.58 & {$[1.57,1.58]$} & 0.98 & {$[0.55,1.24]$} \\
4 & 4.01 & {$[3.83,4.01]$} & 0.44 & {$[0.44,1.40]$} \\
5 & 5.02 & {$[5.00,5.02]$} & 0.32 & {$[0.06,1.96]$} \\
\hline
\end{tabular}

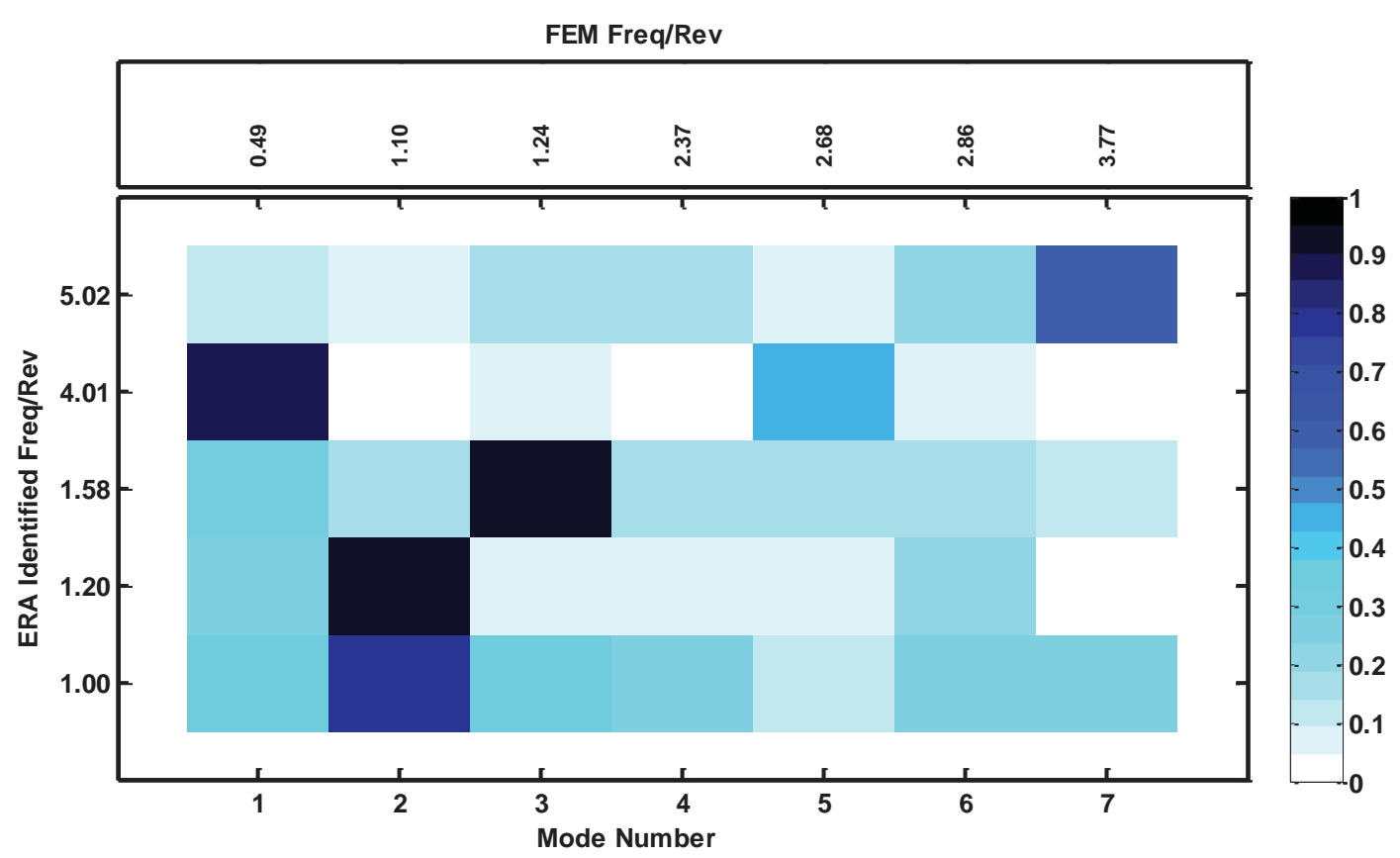

Figure 30. Orthogonality of identified modes versus the finite element model.

\section{Summary and Conclusions}

Our detailed analytical and experimental investigations into the coupled structural dynamics of heliogyro membrane blades have been encouraging and, to date, have revealed no intractable stability and control issues for the heliogyro solar sail concept. Significant accomplishments include:

- Development of a coupled nonlinear finite element heliogyro structural dynamics modeling capability including solar radiation pressure;

- Development of a computationally efficient analytical solarelasticity modeling capability useful for heliogyro stability studies, including stability during blade deployment;

- Development of a small-scale heliogyro dynamics ground testing capability.

- Development of an output-only system identification procedure suitable for on-orbit heliogyro blade synamics investigations.

Furthermore, we conclude that:

- Free-spinning heliogyro systems do not appear to encounter certain solarelastic stabilities predicted using constant-spin rate models; 
- Nonlinear torsional instabilities predicted for high cyclic pitch control amplitudes may be mitigated by redirecting centrifugal tension loading to the leading and trailing edges of the blades, or by adding tip mass to increase centrifugal stiffening.

Ultimately, an actual spaceflight validation mission will be needed to prove feasibility of the heliogyro solar sail concept and retire risk. Given a near term effort to advance the technology readiness of critical systems, most notably blade dynamics simulation capabilities, deployment mechanisms, solarelastic flutter dynamics and control systems including damping augmentation, and relevant ground test demonstrations, a low-cost heliogyro solar sail flight demonstration appears feasible in the near future.

\section{Acknowledgements}

The authors wish to thank the following individuals for their assistance in conducting this work: Doug Lisman, Phil Walkemeyer, Vinh Bach and Mark Thomson, at JPL; Andy Heaton, at NASA Marshal Space Flight Center; Justin Littell, Christopher Savage, Wayne Matthews and Thomas Walker, at NASA Langley Research Center; Thomas Cleckner and Brittany Gater, at Virginia Tech; Andrew Napiecek, at the University of Virginia; Dadija Bliudzius, at Embrey-Riddle Aeronautical Univeristy-Prescott; John Thomson, at University of California, San Diego; Paige Nardozzo, at Virginia Military Institute; and Stephanie Lopez, at University of Puerto Rico.

\section{References}

1. MacNeal, R., “The Heliogyro: An Interplanetary Flying Machine,” NASA Contractor Report CR 84460, June 1967.

2. MacNeal, R., Hedgepeth, J., "Helicopters for Interplanetary Space Flight," 34th National Forum of the American Helicopter Society, Washington, D. C., May 1978.

3. Mori, O., et al., "Overview of IKAROS Mission," in: Macdonald, M. (Ed.), Advances in Solar Sailing, pp. 25-43, SpringerPraxis, Springer Berlin Heidelberg, 2014.

4. Wilkie, W., et al., "Heliogyro Solar Sail Research at NASA," in: Macdonald, M. (Ed.), Advances in Solar Sailing, pp. 631650, Springer-Praxis, Springer Berlin Heidelberg, 2014.

5. Blomquist, R., "Design Study of a Solid-State Heliogyro Solar Sail," M.S. thesis, Massachusetts Institute of Technology, Cambridge, MA, September 1990.

6. McInnes, C., Solar Sailing: Technology, Dynamics and Mission Applications, 1st ed., Springer-Praxis, Chichester, UK, 1999.

7. Macdonald, M., McInnes, C., "Solar Sail Science Mission Applications and Advancement," Advances in Space Research, 48 (2011) 1702-1716.

8. MacNeal, R., "Structural Dynamics of the Heliogyro," NASA CR-17445A, 1971.

9. Dassault Systèmes Simulia Corp., Providence, RI, USA.

10. Blevins, R. D., Formulas for Natural Frequency and Mode Shape, Reprint Edition, Krieger Publishing Company, Malabar, FL, 2001.

11. Blomquist, R., "Heliogyro Control," Ph.D. dissertation, The Robotics Institute, Carnegie Mellon University, Pittsburgh, PA, 2009.

12. Dowell, E., “Can Solar Sails Flutter?,” AIAA Journal, Vol. 49 (2011) 1305-1307.

13. Gibbs, S., Dowell, E., "Solarelastic Stability of the Heliogyro," in: Macdonald, M. (Ed.), Advances in Solar Sailing, pp. 661 665, Springer-Praxis, Springer Berlin Heidelberg, 2014.

14. Natori, M., Nemat-Nasser, S., Mitsugi, J., "Instability of a Rotating Blade Subjected to Solar Radiation Pressure," AIAA 30th Structures, Structural Dynamics and Materials Conference, April 1989.

15. Gibbs, Samuel C., 'Stability of Beams, Plates and Membranes Due to Subsonic Aerodynamic Flows and Solar Radiation Pressure," Ph.D dissertation, Duke University, Durham, NC, 2014.

16. Guerrant, D., D. Lawrence, W. K. Wilkie. "Performance of a Heliogyro Blade Twist Controller with Finite Bandwidth," AIAA/AAS Astrodynamics Specialist Conference, August 2012, Minneapolis, MN.

17. Guerrant, D. and D. Lawrence, "Heliogyro Solar Sail Blade Twist Stability Analysis of Root and Reflectivity Controllers," AIAA Guidance, Navigation and Control Conference, August 2012, Minneapolis, MN.

18. Guerrant, D., Lawrence, D., "Nonlinear Torsional Dynamics and Control of Heliogyro Solar Sail Blades," 2nd AIAA Spacecraft Structures Conference, Kissimmee, FL, 5-9 January 2015.

19. MacNeal, R., Hedgepeth, J., "Helicopters for Interplanetary Space Flight," 34th National Forum of the American Helicopter Society, Washington, D. C., May 1978.

20. "Solar Sail Technology Readiness Report," 720-1, Jet Propulsion Laboratory, July 1977.

21. Wilkie, W., Warren, J., Horta, L. Juang, J., Gibbs, S., Dowell, E., Guerrant, D., Lawrence, D., "Recent Progress in Heliogyro Solar Sail Structural Dynamics," presented at the 2014 European Conference on Spacecraft Structures, Materials, and Environmental Testing, Braunschweig, Germany, April 1-4, 2014.

22. Juang, J.-N., Horta, L., Phan, M., "System/Observer/Controller Identification Toolbox,” NASA TM 107566, February 1992. 